\title{
Online Regularized Classification Algorithms
}

\author{
Yiming Ying and Ding-Xuan Zhou
}

\begin{abstract}
This paper considers online classification learning algorithms based on regularization schemes in reproducing kernel Hilbert spaces associated with general convex loss functions. A novel capacity independent approach is presented. It verifies the strong convergence of the algorithm under a very weak assumption of the step sizes and yields satisfactory convergence rates for polynomially decaying step sizes. Explicit learning rates with respect to the misclassification error are given in terms of the choice of step sizes and the regularization parameter (depending on the sample size). Error bounds associated with the hinge loss, the least square loss, and the support vector machine $q$-norm loss are presented to illustrate our method.
\end{abstract}

Index Terms-Classification algorithm, online learning, reproducing kernel Hilbert spaces, regularization, error analysis.

\section{INTRODUCTION}

$\mathbf{I}$ $\mathrm{N}$ this paper we study online classification algorithms generated from Tikhonov regularization schemes associated with general convex loss functions and reproducing kernel Hilbert spaces.

Let $X$ be a compact metric space and $Y=\{1,-1\}$. A function $\mathcal{C}: X \rightarrow Y$ is called a (binary) classifier which divides the input space $X$ into two classes. A real valued function $f: X \rightarrow \mathbb{R}$ can be used to generate a classifier $\mathcal{C}(x)=\operatorname{sgn}(f(x))$ where the sign function is defined as $\operatorname{sgn}(f(x))=1$ if $f(x) \geq 0$ and $\operatorname{sgn}(f(x))=-1$ for $f(x)<0$. For such a real valued function $f$, a loss function $\phi: \mathbb{R} \rightarrow \mathbb{R}_{+}$ is often used to measure the error: $\phi(y f(x))$ is the local error at the point $(x, y)$ while $\operatorname{sgn}(f(x)) \in Y$ is assigned to the event $x \in X$.

Reproducing Kernel Hilbert spaces are often used as hypothesis spaces in the design of classification algorithms. Let $K: X \times X \rightarrow \mathbb{R}$ be continuous, symmetric and positive semidefinite, i.e., for any finite set of distinct points $\left\{x_{1}, \cdots, x_{\ell}\right\} \subset X$, the matrix $\left(K\left(x_{i}, x_{j}\right)\right)_{i, j=1}^{\ell}$ is positive semidefinite. Such a function is called a Mercer kernel.

The Reproducing Kernel Hilbert Space (RKHS) $\mathcal{H}_{K}$ associated with the kernel $K$ is defined [2] to be the completion of the linear span of the set of functions $\left\{K_{x}=K(x, \cdot)\right.$ : $x \in X\}$ with the inner product $\langle\cdot, \cdot\rangle_{K}$ given by $\left\langle K_{x}, K_{y}\right\rangle_{K}=$ $K(x, y)$. The reproducing property takes the form

$$
\left\langle K_{x}, f\right\rangle_{K}=f(x), \quad x \in X, \quad f \in \mathcal{H}_{K} .
$$

Manuscript received May 11, 2005; revised October 26, 2005. This work was supported by a grant from the Research Grants Council of Hong Kong [Project No. CityU 103704] and by a grant from City University of Hong Kong [Project No. 7001816].

Both Yiming Ying and Ding-Xuan Zhou are with the Department of Mathematics, City University of Hong Kong, Kowloon, Hong Kong, CHINA The corresponding author is Ding-Xuan Zhou (email: mazhou@cityu.edu.hk). Yiming Ying is on leave from Institute of Mathematics, Chinese Academy of Sciences. He is currently with the Department of Computer Science, University College London, London, UK (email: y.ying@cs.ucl.ac.uk).

Communicated by P. L. Bartlett, Associate Editor for Pattern Recognition, Statistical Learning and Inference.
Classification algorithms considered here are induced by regularization schemes learned from samples. Assume that $\rho$ is a probability distribution on $Z=X \times Y$ and $\mathbf{z}=\left\{z_{t}=\right.$ $\left.\left(x_{t}, y_{t}\right)\right\}_{t=1}^{T} \in Z^{T}$ is a set of random samples independently drawn according to $\rho$. The batch learning algorithm for classification is implemented by an off-line regularization scheme [29] in the RKHS $\mathcal{H}_{K}$ involving the sample $\mathbf{z}, \lambda>0$ and the loss function $\phi$ as

$$
f_{\mathbf{z}, \lambda}=\arg \min _{f \in \mathcal{H}_{K}}\left\{\frac{1}{T} \sum_{t=1}^{T} \phi\left(y_{t} f\left(x_{t}\right)\right)+\frac{\lambda}{2}\|f\|_{K}^{2}\right\} .
$$

The classifier $\operatorname{sgn}\left(f_{\mathbf{z}, \lambda}\right)$ is induced by the real valued function $f_{\mathbf{z}, \lambda}$.

The off-line algorithm induced by (2), a Tikhonov regularization scheme for learning [14], has been extensively studied in the literature. In particular, the error analysis is well done due to many results. See e.g. [27], [5], [35], [4], [7], [21], [31], [26]. The main idea of the analysis is to show that $f_{\mathbf{z}, \lambda}$ has behaviors similar to the regularizing function $f_{\lambda} \in \mathcal{H}_{K}$ of scheme (2) defined by

$$
f_{\lambda}=\arg \inf _{f \in \mathcal{H}_{K}}\left\{\mathcal{E}(f)+\frac{\lambda}{2}\|f\|_{K}^{2}\right\} .
$$

Here $\mathcal{E}(f)$ is the generalization error defined as

$$
\mathcal{E}(f)=\int_{Z} \phi(y f(x)) d \rho
$$

This expectation of the similarity between $f_{\mathbf{z}, \lambda}$ and $f_{\lambda}$ is motivated by the law of large numbers telling us that $\frac{1}{T} \sum_{t=1}^{T} \phi\left(y_{t} f\left(x_{t}\right)\right) \rightarrow \mathcal{E}(f)$ with confidence for any fixed function $f$. For a function set, such as the union of unit balls of reproducing kernel Hilbert spaces associated with a set of Mercer kernels, the theory of uniform convergence is involved. See e.g. [29], [1], [34].

Though the off-line algorithm (2) performs well in theory and in many applications, it might be practically challenging when the sample size $T$ or data is very large. For example, when we consider the case $\phi(x)=(1-x)_{+}=\max \{1-x, 0\}$ or $(1-x)_{+}^{2}$ corresponding to the support vector machines, the scheme (2) is a quadratic optimization problem. Its standard complexity is about $O\left(T^{3}\right)$. When $T \geq 10,000$, the algorithm is hard to implement.

Online algorithms with linear complexity $O(T)$ can be applied and provide efficient classifiers, when the sample size is large. In this paper we investigate online classification algorithms generated by Tikhonov regularization schemes in reproducing kernel Hilbert spaces. Convergence to $f_{\lambda}$ in the RKHS norm as well as with respect to the excess misclassification error (to be defined) will be shown, and rate analysis will be done. These online algorithms can be considered as a descent method. See the discussion in the appendix. 
Throughout the paper we assume that the loss function has the following form.

Definition 1: We say that $\phi: \mathbb{R} \longrightarrow \mathbb{R}_{+}$is an admissible loss function if it is convex and differentiable at 0 with $\phi^{\prime}(0)<$ 0 .

The convexity of $\phi$ tells us that the left derivative $\phi_{-}^{\prime}(x)=$ $\lim _{\delta \rightarrow 0-}(\phi(x+\delta)-\phi(x)) / \delta$ exists and equals $\sup _{\delta<0}(\phi(x+$ $\delta)-\phi(x)) / \delta$. It is the same as $\phi^{\prime}(x)$ when it coincides with the right derivative $\phi_{+}^{\prime}(x)=\lim _{\delta \rightarrow 0+}(\phi(x+\delta)-\phi(x)) / \delta=$ $\inf _{\delta>0}(\phi(x+\delta)-\phi(x)) / \delta$. This paper is aimed at the following online algorithm for classification given in [9], [22], [17].

Definition 2: The Stochastic Gradient Descent Online Algorithm for classification is defined by $f_{1}=0$ and

$f_{t+1}=f_{t}-\eta_{t}\left\{\phi_{-}^{\prime}\left(y_{t} f_{t}\left(x_{t}\right)\right) y_{t} K_{x_{t}}+\lambda f_{t}\right\}$ for $t=1, \cdots, T$,

where $\lambda=\lambda(T)>0$ is the regularization parameter and $\eta_{t}>0$ is called the step size. The classifier is given by the sign function $\operatorname{sgn}\left(f_{T+1}\right)$.

We call the sequence $\left\{f_{t}\right\}$ the learning sequence for the online scheme (4) which will be used to learn the regularizing function $f_{\lambda}$.

There is a vast literature on online learning. Let us mention some works relating to this paper. In [22], a stochastic gradient method in the Hilbert space $\mathcal{H}_{K}$ is considered. Let $S L\left(\mathcal{H}_{K}\right)$ be the space of positive definite linear operators on $\mathcal{H}_{K}$, and $A: Z \rightarrow S L\left(\mathcal{H}_{K}\right)$ and $B: Z \rightarrow \mathcal{H}_{K}$ be two maps. To learn a stationary point $f^{*}$ satisfying

$$
\mathbb{E}_{z \in Z}(A(z)+B(z)) f^{*}=0,
$$

they proposed the learning sequence

$$
f_{t+1}=f_{t}-\eta_{t}\left\{A\left(z_{t}\right)\left(f_{t}\right)+B\left(z_{t}\right)\right\} .
$$

But the online scheme (4) involving the general loss function $\phi$ is nonlinear and is hard to write in the setting (5) except for the least square loss. When $\phi$ is the least square loss, we shall improve the estimates in [22] for online regression by presenting capacity independent learning rates (Theorem 3 in Section II), comparable to the best ones in [36], [26] for the off-line regularization setting (2). This is a byproduct of our analysis for online classification algorithms.

The cumulative loss $\frac{1}{T} \sum_{t=1}^{T} \phi\left(y_{t} f_{t}\left(x_{t}\right)\right)$ for online algorithms more general than (4) has been well studied in the literature. See, for example, [15], [3], [10], [9], [16] and references therein. In particular, cumulative loss bounds are derived for online density estimation in [3] and for online linear regression with least square loss in [9]. In Section 6 of [15], for a learning algorithm different from (4), the relative expected instantaneous loss, measuring the prediction ability of $f_{T+1}$ in linear regression problem, is analyzed in detail.

A general regularized online learning scheme (4) is introduced and analyzed in [17]. Assume the loss function $\phi$ is convex, uniformly Lipschitz continuous, the step size has the form $\eta_{t}=O\left(t^{-1 / 2}\right)$, and $\lambda>0$ is fixed. It was proved there that the average instantaneous risk $\frac{1}{T} \sum_{t=1}^{T}\left(\phi\left(y_{t} f_{t}\left(x_{t}\right)\right)+\frac{\lambda}{2}\left\|f_{t}\right\|_{K}^{2}\right)$ converges toward the regularized generalization error $\mathcal{E}\left(f_{\lambda}\right)+$ $\frac{\lambda}{2}\left\|f_{\lambda}\right\|_{K}^{2}$ with error bound $O\left(T^{-1 / 2}\right)$.
In this paper we mainly analyze the error $\left\|f_{T+1}-f_{\lambda}\right\|_{K}$ in the $\mathcal{H}_{K}$ norm, which is different from estimating the cumulative loss bounds as done in many previous results (e.g., [3], [10], [17]). Such a strong approximation was considered for least square regression in [25].

\section{MAIN Results}

The purpose of this paper is to give error bounds for $\left\|f_{T+1}-f_{\lambda}\right\|_{K}$ with fixed $\lambda>0$, and then apply them to the analysis of the online classification algorithm (4): estimating the misclassification error, a quantity measuring the prediction ability, by suitable choices of the regularization parameter $\lambda=\lambda(T)$.

Our first main result states that the sequence $\left\{f_{t}\right\}$ defined by (4) converges in expectation to $f_{\lambda}$ in $\mathcal{H}_{K}$ as long as the sequence is uniformly bounded.

Theorem 1: Let $\lambda>0$ and the sequence of positive step sizes $\left\{\eta_{t}\right\}$ satisfy

$$
\sum_{t=1}^{\infty} \eta_{t}=+\infty, \quad \lim _{t \rightarrow \infty} \eta_{t}=0
$$

If the learning sequence $\left\{f_{t}\right\}$ given in (4) is uniformly bounded in $\mathcal{H}_{K}$, then

$$
\mathbb{E}_{\mathbf{z} \in Z^{T}}\left(\left\|f_{T+1}-f_{\lambda}\right\|_{K}\right) \rightarrow 0 \quad \text { as } T \rightarrow+\infty .
$$

Theorem 1 will be proved in Section V. The assumption of uniform boundedness is mild and will be studied in Section III. In particular, we shall verify that $\left\|f_{t}\right\|_{K}$ is uniformly bounded when $\sup _{|x| \leq R}\left|\phi_{-}^{\prime}(x)-\phi^{\prime}(0)\right| /|x|<\infty$ for any $R$ and $\eta_{t} \leq$ $c_{\lambda}$ for some constant $c_{\lambda}$.

Recall from (3) that $f_{\lambda}$ is the minimizer of the regularized generalization error

$$
\mathcal{E}(f)+\frac{\lambda}{2}\|f\|_{K}^{2}
$$

Our second main result is the following important relation between the excess regularized generalization error and the $\mathcal{H}_{K}$ metric which plays an essential role in proving Theorem 1.

Theorem 2: Let $\phi$ be an admissible loss function and $\lambda>0$. For any $f \in \mathcal{H}_{K}$, there holds

$$
\frac{\lambda}{2}\left\|f-f_{\lambda}\right\|_{K}^{2} \leq\left\{\mathcal{E}(f)+\frac{\lambda}{2}\|f\|_{K}^{2}\right\}-\left\{\mathcal{E}\left(f_{\lambda}\right)+\frac{\lambda}{2}\left\|f_{\lambda}\right\|_{K}^{2}\right\}
$$

Theorem 2 will be proved in Section IV. It will be used to derive convergence rates of $\mathbb{E}_{\mathbf{z} \in Z^{T}}\left(\left\|f_{T+1}-f_{\lambda}\right\|_{K}\right)$ (more quantitative than (7)) when the step size decays in the form $\eta_{t}=t^{-\theta} / \mu(\lambda)$ with a constant $\mu(\lambda)$ (see Theorem 5 in Section VI). These rates, together with the approximation error (called the regularization error below) between $f_{\lambda}$ and the minimizer of the generalization error, yield capacity independent learning rates of the misclassification error of the online algorithm (4). This is our last main result illustrated in the following subsections. 
TABLE I

NOTATIONS

\begin{tabular}{|l|l|l|}
\hline notation & meaning & pages \\
\hline \hline$\phi$ & loss function for classification & 1,2 \\
\hline$\lambda$ & regularization parameter & 1,2 \\
\hline$f_{\mathbf{z}, \lambda}$ & off-line algorithm $(2)$ & 1 \\
\hline $\mathcal{E}$ & generalization error & 1 \\
\hline$f_{\lambda}$ & regularizing function & 1 \\
\hline$\eta_{t}$ & step size & $2,2,12$ \\
\hline $\mathcal{R}$ & misclassification error & 3 \\
\hline$f_{c}$ & Bayes rule & 3 \\
\hline$f_{\rho}$ & regression function & 3 \\
\hline $\mathbb{E}$ & expectation wrt $\left(z_{1}, \ldots, z_{T}\right) \in Z^{T}$ & 3 \\
\hline$\kappa$ & the constant sup $x \in X \sqrt{K(x, x)}$ & 3 \\
\hline$L_{K}$ & integral operator with kernel $K$ & 3,11 \\
\hline$f_{\rho}^{\phi}$ & minimizer of $\mathcal{E}$ with loss $\phi$ & 4 \\
\hline $\mathcal{D}(\lambda)$ & regularization error & 4 \\
\hline$M(\lambda)$ & local Lipschitz constant & 5 \\
\hline$\widetilde{C}_{\lambda}>0$ & constant measuring the increment of $\phi_{-}^{\prime}$ & 8,9 \\
\hline $\mathbb{E}_{z_{1}, \ldots, z_{t}}$ & expectation wrt $z_{1}, \ldots, z_{t}$ & 8 \\
\hline$\mu(\lambda)$ & constant for the step size $\eta_{t}=\frac{1}{\mu(\lambda) t^{\theta}}$ & 9,10 \\
\hline \hline
\end{tabular}

\section{A. Misclassification error of classification algorithms}

The prediction power of classification algorithms are often measured by the misclassification error which is defined for a classifier $\mathcal{C}: X \rightarrow Y$ to be the probability of the event $\{\mathcal{C}(x) \neq y\}$ :

$$
\mathcal{R}(\mathcal{C})=\operatorname{Prob}\{\mathcal{C}(x) \neq y\}=\int_{X} P(y \neq \mathcal{C}(x) \mid x) d \rho_{X} .
$$

Here $\rho_{X}$ denotes the marginal distribution of $\rho$ on $X$, and $P(\cdot \mid x)$ the conditional probability measure. The best classifier minimizing the misclassification error is called the Bayes rule [13] and can be expressed as $f_{c}=\operatorname{sgn}\left(f_{\rho}\right)$ where $f_{\rho}$ is the regression function

$$
f_{\rho}(x)=\int_{Y} y d \rho(y \mid x)=P(y=1 \mid x)-P(y=-1 \mid x) .
$$

Recall that for the online learning algorithm (4), we are interested in the classifier $\operatorname{sgn}\left(f_{T+1}\right)$ produced by the real valued function $f_{T+1}$ from a sample $\mathbf{z}=\left\{z_{t}\right\}_{t=1}^{T}$. So the error analysis for the classification algorithm (4) is aimed at the excess misclassification error

$$
\mathcal{R}\left(\operatorname{sgn}\left(f_{T+1}\right)\right)-\mathcal{R}\left(f_{c}\right) .
$$

Let us present some examples proved in Section VII to illustrate the learning rates of (12) from suitable choices of the regularization parameter $\lambda=\lambda(T)$ and the step size $\eta_{t}$.

The first example corresponds to the classical support vector machine (SVM) with $\phi$ being the hinge loss $\phi(x)=(1-x)_{+}$. For this loss, the online algorithm (4) can be expressed as $f_{1}=0$ and

$$
f_{t+1}= \begin{cases}\left(1-\eta_{t} \lambda\right) f_{t}, & \text { if } y_{t} f_{t}\left(x_{t}\right)>1 \\ \left(1-\eta_{t} \lambda\right) f_{t}+\eta_{t} y_{t} K_{x_{t}}, & \text { if } y_{t} f_{t}\left(x_{t}\right) \leq 1\end{cases}
$$

Corollary 1: Let $\phi(x)=(1-x)_{+}$. Assume for some $0<$ $\beta \leq 1$, the pair $(\rho, K)$ satisfies

$$
\inf _{f \in \mathcal{H}_{K}}\left\{\left\|f-f_{c}\right\|_{L_{\rho_{X}}^{1}}+\lambda\|f\|_{K}^{2}\right\}=O\left(\lambda^{\beta}\right) .
$$

For any $0<\varepsilon<\frac{\beta}{2(\beta+1)}$, choose $\lambda=\lambda(T)=T^{\frac{\varepsilon}{\beta}-\frac{1}{2(\beta+1)}}$ and $\eta_{t}=\frac{1}{2+\kappa^{2}} t^{\frac{(2 \beta+1) \varepsilon}{\beta}-\frac{2 \beta+1}{2(\beta+1)}}$ where $\kappa=\sup _{x \in X} \sqrt{K(x, x)}$. Then

$$
\mathbb{E}\left(\mathcal{R}\left(\operatorname{sgn}\left(f_{T+1}\right)\right)-\mathcal{R}\left(f_{c}\right)\right)=O\left(T^{\varepsilon-\frac{\beta}{2(\beta+1)}}\right) .
$$

In (15), the expectation $\mathbb{E}$ is taken with respect to the random sample $\mathbf{z} \in Z^{T}$. We shall use this notion throughout the paper, if the random variable for $\mathbb{E}$ is not specified.

The condition (14) concerns the approximation of the function $f_{c}$ in the $L^{1}$ space $L_{\rho_{X}}^{1}$ by functions from the RKHS $\mathcal{H}_{K}$. It can be characterized by requiring $f_{c}$ to lie in an interpolation space of the pair $\left(L_{\rho_{X}}^{1}, \mathcal{H}_{K}\right)$, an intermediate space between the metric space $L_{\rho_{X}}^{1}$ and the much smaller approximating space $\mathcal{H}_{K}$. For details, see the discussion in [7]. The assumption (14) is satisfied [34] when we use the Gaussian kernels with flexible variances and the distribution satisfies some geometric noise condition [21].

Assumptions like (14) are necessary to determine the regularization parameter for achieving the learning rate (15). This can be seen from the literature [21], [31], [35] of off-line algorithm (2): learning rates are obtained by suitable choices of the regularization parameter $\lambda=\lambda(T)$, according to the behavior of the approximation error estimated from a priori conditions on the distribution $\rho$ and the space $\mathcal{H}_{K}$.

The second example is for the least square loss. Here the approximation error [23], [25] can be studied by the integral operator $L_{K}: L_{\rho_{X}}^{2} \rightarrow L_{\rho_{X}}^{2}$ on the $L^{2}$ space $L_{\rho_{X}}^{2}$ defined by

$$
L_{K}(f)(x)=\int_{X} K(x, y) f(y) d \rho_{X}, \quad x \in X, f \in L_{\rho_{X}}^{2} .
$$

Since $K$ is a Mercer kernel, the operator $L_{K}$ is symmetric, compact and positive. Therefore its power $L_{K}^{\beta}$ is well-defined for any $0<\beta \leq 1$.

Corollary 2: Let $\phi(x)=(1-x)^{2}$ and $f_{\rho}$ be in the range of $L_{K}^{\beta}$ with some $0<\beta \leq 1$. For any $0<\varepsilon<\frac{\beta}{2(\beta+1)}$, take $\lambda=\lambda(T)=T^{\frac{\varepsilon}{\beta}-\frac{1}{2(\beta+1)}}$ and $\eta_{t}=\frac{1}{8 \kappa^{2}+\lambda} t^{\frac{(2 \beta+1) \varepsilon}{\beta}-\frac{2 \beta+1}{2(\beta+1)}}$. Then

$$
\begin{aligned}
\mathbb{E}\left(\mathcal{R}\left(\operatorname{sgn}\left(f_{T+1}\right)\right)-\mathcal{R}\left(f_{c}\right)\right) & \leq \mathbb{E}\left(\left\|f_{T+1}-f_{\rho}\right\|_{L_{\rho_{X}}^{2}}\right) \\
& =O\left(T^{\varepsilon-\frac{\beta}{2(\beta+1)}}\right)
\end{aligned}
$$

Let us analyze the assumption as in [25]. Denote $\left\{\lambda_{i}\right\}_{i \geq 1}$ as the positive eigenvalues of the positive, compact operator $L_{K}$ and $\left\{\varphi_{i}\right\}_{i \geq 1}$ the corresponding orthonormal eigenfunctions. Any function $f \in L_{\rho_{X}}^{2}$ can be expressed as $f=\sum_{i \geq 1} c_{i} \varphi_{i}+$ $f_{0}$ with $\left\{c_{i}\right\} \in \ell^{2}$ and $f_{0}$ satisfying $L_{K} f_{0}=0$. The function $f$ lies in the range of $L_{K}^{\beta}$ for some $0<\beta \leq 1$ if and only if $\left\{\lambda_{i}^{-\beta} c_{i}\right\} \in \ell^{2}$, that is, the sequence $\left\{c_{i}\right\}$ has such a nice decay that $c_{i}=\lambda_{i}^{\beta} d_{i}$ for another $\ell^{2}$ sequence $\left\{d_{i}\right\}$. In particular [11], $f$ is in the range of $L_{K}^{1 / 2}$ if and only if $f \in \mathcal{H}_{K}$. So the smaller $\beta$ is, the less demanding the assumption in Corollary 2 is. When $\beta \leq 1 / 2$, this assumption can also be characterized [23] by the decay $\inf _{f \in \mathcal{H}_{K}}\left\{\left\|f-f_{\rho}\right\|_{L_{\rho_{X}}^{2}}^{2}+\frac{\lambda}{2}\|f\|_{K}^{2}\right\}=O\left(\lambda^{2 \beta}\right)$. For the least square loss, there holds $\left\|f-f_{\rho}\right\|_{L_{\rho_{X}}^{2}}^{2}=\mathcal{E}(f)-$ $\mathcal{E}\left(f_{\rho}\right)$. This leads to the condition on the regularization error discussed in the next subsection for the general loss. 


\section{B. Comparison theorem and regularization error}

Estimating excess misclassification error $\mathcal{R}\left(\operatorname{sgn}\left(f_{T+1}\right)\right)-$ $\mathcal{R}\left(f_{c}\right)$ in (12) can often be done by bounding the excess generalization error [35], [4], [7]

$$
\mathcal{E}\left(f_{T+1}\right)-\mathcal{E}\left(f_{\rho}^{\phi}\right)
$$

where $f_{\rho}^{\phi}$ is a minimizer of the generalization error

$$
f_{\rho}^{\phi}=\arg \inf \{\mathcal{E}(f): f \text { is measurable on } X\} .
$$

In particular for the SVM 1-norm soft margin classifier with the hinge loss $\phi(x)=(1-x)_{+}$, we have $f_{\rho}^{\phi}=f_{c}$ [30] and an important relation was given in [35] as

$$
\mathcal{R}(\operatorname{sgn}(f))-\mathcal{R}\left(f_{c}\right) \leq \mathcal{E}(f)-\mathcal{E}\left(f_{c}\right) .
$$

Such a relation is called a comparison theorem. For the general loss function, a simple comparison theorem was established in [7], [4].

Proposition A: Let $\phi$ be an admissible loss function such that $\phi^{\prime \prime}(0)$ exists and is positive. Then there is a constant $c_{\phi}$ such that for any measurable function $f$, there holds

$$
\mathcal{R}(\operatorname{sgn}(f))-\mathcal{R}\left(f_{c}\right) \leq c_{\phi} \sqrt{\mathcal{E}(f)-\mathcal{E}\left(f_{\rho}^{\phi}\right)} .
$$

If moreover, for some $\tau \in[0,1]$ and $c>0, \rho$ satisfies a Tsybakov noise condition: for any measurable function $f$,

$$
\rho_{X}\left(\operatorname{sgn}(f) \neq f_{c}\right) \leq c\left\{\mathcal{R}(\operatorname{sgn}(f))-\mathcal{R}\left(f_{c}\right)\right\}^{\tau},
$$

then (19) can be improved as

$$
\mathcal{R}(\operatorname{sgn}(f))-\mathcal{R}\left(f_{c}\right) \leq\left\{2 c_{\phi} c\left(\mathcal{E}(f)-\mathcal{E}\left(f_{\rho}^{\phi}\right)\right)\right\}^{1 /(2-\tau)} .
$$

The Tsybakov noise condition (20) was introduced in [28] where the reader can find more details and explanation. The greater $\tau$ is, the smaller the noise of $\rho$ is. In particular, any distribution $\rho$ satisfies (20) with $\tau=0$ and $c=1$.

With a comparison theorem, it is sufficient for us to estimate the excess generalization error (17). In order to do so, we need the regularization error [24] between $f_{\lambda}$ and $f_{\rho}^{\phi}$.

Definition 3: The regularization error for (2) associated with the triple $(K, \phi, \rho)$ is

$$
\begin{aligned}
\mathcal{D}(\lambda) & =\inf _{f \in \mathcal{H}_{K}}\left\{\mathcal{E}(f)-\mathcal{E}\left(f_{\rho}^{\phi}\right)+\frac{\lambda}{2}\|f\|_{K}^{2}\right\} \\
& =\mathcal{E}\left(f_{\lambda}\right)-\mathcal{E}\left(f_{\rho}^{\phi}\right)+\frac{\lambda}{2}\left\|f_{\lambda}\right\|_{K}^{2}, \quad \lambda>0 .
\end{aligned}
$$

Now we have the error decomposition [32] for (17) as

$$
\begin{aligned}
\mathcal{E}\left(f_{T+1}\right)-\mathcal{E}\left(f_{\rho}^{\phi}\right) & =\mathcal{E}\left(f_{T+1}\right)-\mathcal{E}\left(f_{\lambda}\right)+\mathcal{E}\left(f_{\lambda}\right)-\mathcal{E}\left(f_{\rho}^{\phi}\right) \\
& \leq \mathcal{E}\left(f_{T+1}\right)-\mathcal{E}\left(f_{\lambda}\right)+\mathcal{D}(\lambda) .
\end{aligned}
$$

The regularization error term $\mathcal{D}(\lambda)$ in the error decomposition (22) is independent of the sample $\mathbf{z}=\left\{z_{t}\right\}_{t=1}^{T}$. It can be estimated by $K$-functionals from the rich knowledge of approximation theory. For more details, see discussions in [23], [7], [31].

The first term $\mathcal{E}\left(f_{T+1}\right)-\mathcal{E}\left(f_{\lambda}\right)$ in (22) is called the sample error which may be bounded by the error $\left\|f_{T+1}-f_{\lambda}\right\|_{K}$. To show the idea, we mention a rough approach here. More refined estimates are possible for specific loss functions as shown in Section VII. Observe from the convexity of $\phi$ that $\phi_{-}^{\prime}$ and $\phi_{+}^{\prime}$ are both nondecreasing, and $\phi_{-}^{\prime}(x) \leq \phi_{+}^{\prime}(x)$.

Denote $C(X)$ as the space of continuous functions on $X$ with the norm $\|\cdot\|_{\infty}$. Then the reproducing property (1) tells us that

$$
\|f\|_{\infty} \leq \kappa\|f\|_{K}, \quad \forall f \in \mathcal{H}_{K}
$$

Corollary 3: Let $\phi$ be an admissible loss function and $f \in$ $\mathcal{H}_{K}$. There holds

$\mathcal{E}(f)-\mathcal{E}\left(f_{\lambda}\right) \leq \kappa\left\|f-f_{\lambda}\right\|_{K} \max \left\{\left\|\phi_{+}^{\prime}\right\|_{L^{\infty}\left(I_{\lambda}\right)},\left\|\phi_{-}^{\prime}\right\|_{L^{\infty}\left(I_{\lambda}\right)}\right\}$

where $I_{\lambda}$ is the interval $I_{\lambda}=\left[-C_{\lambda, f}, C_{\lambda, f}\right]$ with $C_{\lambda, f}:=$ $\max \left\{\kappa\|f\|_{K}, \kappa \sqrt{2 \mathcal{D}(\lambda) / \lambda}\right\}$.

Proof: Since $\mathcal{E}\left(f_{\lambda}\right)-\mathcal{E}\left(f_{\rho}^{\phi}\right) \geq 0$, the definition for $\mathcal{D}(\lambda)$ tells us that

$$
\left\|f_{\lambda}\right\|_{K} \leq \sqrt{2 \mathcal{D}(\lambda) / \lambda}
$$

Note the elementary inequality

$$
|\phi(u)-\phi(s)| \leq \max \left\{\left\|\phi_{+}^{\prime}\right\|_{L^{\infty}(I)},\left\|\phi_{-}^{\prime}\right\|_{L^{\infty}(I)}\right\}|u-s|
$$

where $I$ is an interval containing $u$ and $s$. Applying this inequality with $u=y f(x)$ and $s=y f_{\lambda}(x)$, we know that for any $y \in Y, x \in X,\left|\phi(y f(x))-\phi\left(y f_{\lambda}(x)\right)\right|$ can be bounded by $\left|f(x)-f_{\lambda}(x)\right| \max \left\{\left\|\phi_{+}^{\prime}\right\|_{L^{\infty}(I)},\left\|\phi_{-}^{\prime}\right\|_{L^{\infty}(I)}\right\}$, where $I$ is an interval containing $y f(x)$ and $y f_{\lambda}(x)$. But (23) implies $|y f(x)| \leq\|f\|_{\infty} \leq \kappa\|f\|_{K}$ and $\left|y f_{\lambda}(x)\right| \leq \kappa\|f\|_{K} \leq$ $\kappa \sqrt{2 \mathcal{D}(\lambda) / \lambda}$. In connection with the fact $\mathcal{E}(f)-\mathcal{E}\left(f_{\lambda}\right) \leq$ $\int_{Z}\left|\phi(y f(x))-\phi\left(y f_{\lambda}(x)\right)\right| d \rho$ and the inequality $\left\|f-f_{\lambda}\right\|_{\infty} \leq$ $\kappa\left\|f-f_{\lambda}\right\|_{K}$, we verify the desired bound.

Let us show by the example of SVM $q$-norm loss the learning rates of the excess misclassification error for the online algorithm (4), derived from the regularization error, the choice of the regularization parameter and the step size.

Corollary 4: Let $\phi(x)=(1-x)_{+}^{q}$ with $q>1$. Assume that for some $0<\beta<1$, there holds $\mathcal{D}(\lambda)=O\left(\lambda^{\beta}\right)$. For any $0<\varepsilon<\frac{\beta}{2((2-\beta) q+3 \beta)}$, take $\lambda=T^{-\gamma}$ and $\eta_{t}=$ $\frac{\lambda^{\min \{q-2,0\}}}{\left(1+2 \kappa^{2 q}\right)(2 q)^{q+1}} t^{-\alpha \gamma}$ with

$\gamma=\frac{1}{(2-\beta) q+3 \beta}-\frac{2 \varepsilon}{\beta}, \quad \alpha=\min \{q-1,1\}+(1-\beta) q+3 \beta$.

Then

$$
\mathbb{E}\left(\mathcal{R}\left(\operatorname{sgn}\left(f_{T+1}\right)\right)-\mathcal{R}\left(f_{c}\right)\right)=O\left(T^{\varepsilon-\frac{\beta}{2[(2-\beta) q+3 \beta]}}\right) .
$$

If moreover, the distribution $\rho$ satisfies (20), then the rate can be improved to

$$
\mathbb{E}\left(\mathcal{R}\left(\operatorname{sgn}\left(f_{T+1}\right)\right)-\mathcal{R}\left(f_{c}\right)\right)=O\left(T^{\frac{1}{2-\tau}\left(2 \varepsilon-\frac{\beta}{[(2-\beta) q+3 \beta]}\right)}\right) .
$$

\section{A byproduct for regression and rate comparison}

Our method for the classification algorithm can provide not only estimates for the excess misclassification error, but also estimates for the strong approximation in the $\mathcal{H}_{K}$ metric. Let us show this by the least square loss. We assume that $f_{\rho}$ lies in the range of $L_{K}^{\beta}$ for some $1 / 2<\beta \leq 1$.

Theorem 3: Let $\phi(x)=(1-x)^{2}$ and $f_{\rho}$ be in the range of $L_{K}^{\beta}$ for some $1 / 2<\beta \leq 1$. For any $0<\varepsilon<\frac{\beta}{2(\beta+1)}$, 
take $\lambda=\lambda(T)=T^{\frac{\varepsilon}{\beta}-\frac{1}{2(\beta+1)}}$ and $\eta_{t}=\frac{1}{8 \kappa^{2}+\lambda} t^{\frac{(2 \beta+1) \varepsilon}{\beta}-\frac{2 \beta+1}{2(\beta+1)}}$. Then

$$
\mathbb{E}\left(\left\|f_{T+1}-f_{\rho}\right\|_{K}\right)=O\left(T^{\varepsilon-\frac{2 \beta-1}{4 \beta+2}}\right) .
$$

Let us compare our learning rates for the least square loss with the existing results.

First, we will show that our learning rate in the online setting is comparable to that in the off-line setting under the same assumption on the approximation error: $\left\|L_{K}^{-\beta} f_{\rho}\right\|_{L_{\rho_{X}}^{2}}<\infty$. In [5], [36], a leave-one-out technique was used to derive the expected value of the off-line regularization algorithm (2). The results in [35], [36] can be expressed as

$$
\mathbb{E}\left(\mathcal{E}\left(f_{\mathbf{z}, \lambda}\right)\right) \leq\left(1+\frac{2 \kappa^{2}}{T \lambda}\right)^{2} \inf _{f \in \mathcal{H}_{K}}\left\{\mathcal{E}(f)+\frac{\lambda}{2}\|f\|_{K}^{2}\right\} .
$$

In terms of the regularization error, it can be restated as

$$
\begin{aligned}
& \mathbb{E}\left(\left\|f_{\mathbf{z}, \lambda}-f_{\rho}\right\|_{L_{\rho_{X}}^{2}}^{2}\right) \\
\leq & \mathcal{D}(\lambda)+\left(\mathcal{E}\left(f_{\rho}\right)+\mathcal{D}(\lambda)\right)\left\{\frac{4 \kappa^{2}}{T \lambda}+\left(\frac{2 \kappa^{2}}{T \lambda}\right)^{2}\right\} .
\end{aligned}
$$

We know from Lemma 3 in [26] that if $\left\|L_{K}^{-\beta} f_{\rho}\right\|_{L_{\rho_{X}}^{2}}<\infty$ for some $0<\beta \leq 1 / 2$ then

$$
\mathcal{D}(\lambda) \leq \lambda^{2 \beta}\left\|L_{K}^{-\beta} f_{\rho}\right\|_{L_{\rho_{X}}^{2}} .
$$

Putting this into (26) and trading off $T$ and $\lambda$, we know that the choice $\lambda=T^{-\frac{1}{1+2 \beta}}$ gives the optimal rate

$$
\mathbb{E}\left(\left\|f_{\mathbf{z}, \lambda}-f_{\rho}\right\|_{L_{\rho_{X}}^{2}}\right)=O\left(T^{-\frac{\beta}{2 \beta+1}}\right) .
$$

If $f_{\rho}$ is in the range of $L_{K}^{\beta}$ with $\beta>1 / 2$ and $\lambda=$ $C_{\beta, \kappa} T^{-\frac{1}{1+2 \beta}}$ for some constant $C_{\beta, \kappa}$, the $\mathcal{H}_{K}$ error is given in Theorem 2 of [26] for the off-line regularization scheme (2) as

$$
\mathbb{E}\left(\left\|f_{\mathbf{z}, \lambda}-f_{\rho}\right\|_{K}\right)=O\left(T^{-\frac{2 \beta-1}{4 \beta+2}}\right)
$$

Hence our online learning rate (25) are almost the same as the corresponding (28) in the offline setting while the rate (16) is suboptimal compared with (27).

Next, we will compare our learning rate (16) with the one in [22] under the same assumption: $\left\|L_{K}^{-\beta} f_{\rho}\right\|_{L_{\rho_{X}}^{2}}<\infty$ for some $0<\beta \leq 1$. The result given in Theorem $\mathrm{A}$ and Remark 2.1 of [22] is

$$
\begin{aligned}
& \mathbb{E}\left(\left\|f_{T+1}-f_{\lambda}\right\|_{K}\right) \leq C_{\theta, \kappa}\left(\lambda^{-\frac{1}{2}-\frac{1}{2(1-\theta)}} T^{-\frac{\theta}{2}}\right. \\
& \left.\quad+\exp \left\{-c_{\theta} \lambda T^{1-\theta}\right\}\right) \text { for } \theta \in(1 / 2,1)
\end{aligned}
$$

where $C_{\theta, \kappa}$ and $c_{\theta}$ are constants.

Select $\lambda=T^{-\gamma}$ and $0<\gamma<\frac{\theta(1-\theta)}{2-\theta}$, then there is another constant $\widetilde{C}_{\theta, \kappa}$ such that (29) is rewritten as

$$
\mathbb{E}\left(\left\|f_{T+1}-f_{\lambda}\right\|_{K}\right) \leq \widetilde{C}_{\theta, \kappa} T^{-\left(\frac{\theta}{2}-\frac{(2-\theta)}{2(1-\theta)} \gamma\right)} .
$$

Since $f_{\rho}$ is the range of $L_{K}^{\beta}$ for some $0<\beta \leq 1$, by [26] we have

$$
\left\|f_{\lambda}-f_{\rho}\right\|_{L_{\rho_{X}}^{2}} \leq\left\|L_{K}^{-\beta} f_{\rho}\right\|_{L_{\rho_{X}}^{2}} \lambda^{\beta}=\left\|L_{K}^{-\beta} f_{\rho}\right\|_{L_{\rho_{X}}^{2}} T^{-\beta \gamma} .
$$

Consequently, the choice $\gamma=\frac{\theta(1-\theta)}{(2-\theta)+2 \beta(1-\theta)}$ gives the optimal rate with a constant $C_{\theta, \beta, \kappa}$ :

$$
\mathbb{E}\left(\left\|f_{T+1}-f_{\rho}\right\|_{L_{\rho_{X}}^{2}}\right) \leq C_{\theta, \beta, \kappa} T^{-\frac{\beta \theta(1-\theta)}{(2-\theta)+2 \beta(1-\theta)}} .
$$

Setting $\theta=\frac{2(\beta+1)-\sqrt{2(\beta+1)}}{2 \beta+1}$ and $\gamma=(\sqrt{2(\beta+1)}+1)^{-2}$ gives the optimal rate

$$
\mathbb{E}\left(\left\|f_{T+1}-f_{\rho}\right\|_{L_{\rho_{X}}^{2}}\right) \leq C_{\theta, \beta, \kappa} T^{-\beta(\sqrt{2(\beta+1)}+1)^{-2}} .
$$

Our learning rate (16) is better since $\frac{\beta}{2(\beta+1)}>\beta(\sqrt{2(\beta+1)}+$ $1)^{-2}$.

\section{Bounding THE LEARNING SEQUENCE}

In this section we show how a local Lipschitz condition on the loss function $\phi$ at the origin and some restrictions on the step size $\left\{\eta_{t}\right\}$ ensure the uniform boundedness of the learning sequence $\left\{f_{t}\right\}$, a crucial assumption for the convergence of the online scheme (4) stated in Theorem 1. Hence the uniform boundedness holds for all loss functions encountered in specific classification algorithms.

Definition 4: We say that $\phi_{-}^{\prime}$ is locally Lipschitz at the origin if the local Lipschitz constant

$$
M(\lambda)=\sup \left\{\frac{\left|\phi_{-}^{\prime}(x)-\phi^{\prime}(0)\right|}{|x|}: \quad|x| \leq \frac{\kappa^{2}\left|\phi^{\prime}(0)\right|}{\lambda}\right\}
$$

is finite for any $\lambda>0$.

The above local Lipschitz condition is equivalent to the existence of some $\varepsilon>0$ and $\mathrm{L}>0$ such that $\mid \phi_{-}^{\prime}(x)-$ $\phi^{\prime}(0)|\leq \mathrm{L}| x \mid$ for every $x \in[-\varepsilon, \varepsilon]$. In fact, the latter requirement implies $M(\lambda) \leq \max \left\{\mathrm{L}, \mid \phi_{-}^{\prime}\left(-\kappa^{2}\left|\phi^{\prime}(0)\right| / \lambda\right)-\right.$ $\left.\phi^{\prime}(0) \mid / \varepsilon,\left(\left|\phi_{-}^{\prime}\left(\kappa^{2}\left|\phi^{\prime}(0)\right| / \lambda\right)\right|+\left|\phi^{\prime}(0)\right|\right) / \varepsilon\right\}$. Thus, when $\phi$ is twice continuously differentiable on $\mathbb{R}, \phi_{-}^{\prime}$ is locally Lipschitz at the origin with $M(\lambda)=\left\|\phi^{\prime \prime}\right\|_{L^{\infty}\left[-\kappa^{2}\left|\phi^{\prime}(0)\right| / \lambda, \kappa^{2}\left|\phi^{\prime}(0)\right| / \lambda\right]}$. Examples of loss functions will be discussed after the following theorem on the boundedness of the sequence $\left\{f_{t}\right\}$.

Theorem 4: Assume that $\phi_{-}^{\prime}$ is locally Lipschitz at the origin. Define $\left\{f_{t}\right\}$ by (4). If the step size $\eta_{t}$ satisfies $\eta_{t}\left(M(\lambda) \kappa^{2}+\lambda\right) \leq 1$ for each $t$, then

$$
\left\|f_{t}\right\|_{K} \leq \frac{\kappa\left|\phi^{\prime}(0)\right|}{\lambda}, \quad \forall t \in \mathbb{N} \text {. }
$$

Proof: We prove by induction. It is trivial that $f_{1}=0$ satisfies the bound (32). Suppose that this bound holds true for $f_{t}$. Consider $f_{t+1}$. It can be written as

$\left(1-\eta_{t} \lambda\right) f_{t}-\eta_{t}\left[\phi_{-}^{\prime}\left(y_{t} f_{t}\left(x_{t}\right)\right)-\phi^{\prime}(0)\right] y_{t} K_{x_{t}}-\eta_{t} y_{t} \phi^{\prime}(0) K_{x_{t}}$.

Write the middle term as

$\left[\phi_{-}^{\prime}\left(y_{t} f_{t}\left(x_{t}\right)\right)-\phi^{\prime}(0)\right] y_{t} K_{x_{t}}=\frac{\phi_{-}^{\prime}\left(y_{t} f_{t}\left(x_{t}\right)\right)-\phi^{\prime}(0)}{y_{t} f_{t}\left(x_{t}\right)} L_{t}\left(f_{t}\right)$.

Here by means of the reproducing property (1) for $f_{t}\left(x_{t}\right) K_{x_{t}}=\left\langle f_{t}, K_{x_{t}}\right\rangle_{K} K_{x_{t}}$ we have denoted $L_{t}: \mathcal{H}_{K} \rightarrow$ $\mathcal{H}_{K}$ as a self-adjoint, rank-one, positive linear operator given by

$$
L_{t}(g)=\left\langle g, K_{x_{t}}\right\rangle_{K} K_{x_{t}} .
$$

The operator norm can be bounded as $\left\|L_{t}\right\|_{\mathcal{H}_{K} \rightarrow \mathcal{H}_{K}} \leq \kappa^{2}$ since $\left\langle L_{t} g, g\right\rangle_{K}=\left|\left\langle g, K_{x_{t}}\right\rangle_{K}\right|^{2} \leq \kappa^{2}\|g\|_{K}^{2}$ for any $g \in \mathcal{H}_{K}$. 
The local Lipschitz condition tells us that $\frac{\phi_{-}^{\prime}\left(y_{t} f_{t}\left(x_{t}\right)\right)-\phi^{\prime}(0)}{y_{t} f_{t}\left(x_{t}\right)}$ is well defined (set as zero when $f_{t}\left(x_{t}\right)=0$ ). It is bounded by $M\left(\kappa^{2}\left|\phi^{\prime}(0)\right| / \lambda\right)$, since $\left|y_{t} f_{t}\left(x_{t}\right)\right| \leq \kappa\left\|f_{t}\right\|_{K} \leq \kappa^{2}\left|\phi^{\prime}(0)\right| / \lambda$ by our induction hypothesis. Since $\phi_{-}^{\prime}$ is nondecreasing, $\left(\phi_{-}^{\prime}(u)-\phi^{\prime}(0)\right) / u \geq 0$ for any $u \in \mathbb{R}$. Thus,

$$
0 \leq \frac{\phi_{-}^{\prime}\left(y_{t} f_{t}\left(x_{t}\right)\right)-\phi^{\prime}(0)}{y_{t} f_{t}\left(x_{t}\right)} \leq M\left(\kappa^{2}\left|\phi^{\prime}(0)\right| / \lambda\right) .
$$

Therefore, $\frac{\phi_{-}^{\prime}\left(y_{t} f_{t}\left(x_{t}\right)\right)-\phi^{\prime}(0)}{y_{t} f_{t}\left(x_{t}\right)} L_{t}$ is a self-adjoint, positive linear operator on $\mathcal{H}_{K}$. Its norm is bounded by $\kappa^{2} M\left(\kappa^{2}\left|\phi^{\prime}(0)\right| / \lambda\right)$. When $\eta_{t}\left(M(\lambda) \kappa^{2}+\lambda\right) \leq 1$, the linear operator

$$
\mathcal{A}:=\left(1-\eta_{t} \lambda\right) I-\eta_{t} \frac{\phi_{-}^{\prime}\left(y_{t} f_{t}\left(x_{t}\right)\right)-\phi^{\prime}(0)}{y_{t} f_{t}\left(x_{t}\right)} L_{t}
$$

on $\mathcal{H}_{K}$ is self-adjoint, positive, and $\mathcal{A} \leq\left(1-\eta_{t} \lambda\right) I$. It follows that

$$
\begin{aligned}
& \left\|\left(1-\eta_{t} \lambda\right) f_{t}-\eta_{t}\left[\phi_{-}^{\prime}\left(y_{t} f_{t}\left(x_{t}\right)\right)-\phi^{\prime}(0)\right] y_{t} K_{x_{t}}\right\|_{K} \\
& =\left\|\mathcal{A} f_{t}\right\|_{K} \leq\left\|\mathcal{A}^{1 / 2}\right\|_{\mathcal{H}_{K} \rightarrow \mathcal{H}_{K}}\left\{\left\|\mathcal{A}^{1 / 2} f_{t}\right\|_{K}^{2}\right\}^{1 / 2} \\
& \leq\left(1-\eta_{t} \lambda\right)^{1 / 2}\left\{\left\langle\mathcal{A} f_{t}, f_{t}\right\rangle_{K}\right\}^{1 / 2} \leq\left(1-\eta_{t} \lambda\right)\left\|f_{t}\right\|_{K},
\end{aligned}
$$

since $\left\langle\mathcal{A} f_{t}, f_{t}\right\rangle_{K} \leq\left(1-\eta_{t} \lambda\right)\left\|f_{t}\right\|_{K}^{2}$. This in connection with the induction hypothesis on $\left\|f_{t}\right\|_{K}$ implies that

$$
\left\|f_{t+1}\right\|_{K} \leq\left(1-\eta_{t} \lambda\right) \frac{\kappa\left|\phi^{\prime}(0)\right|}{\lambda}+\kappa \eta_{t}\left|\phi^{\prime}(0)\right|=\frac{\kappa\left|\phi^{\prime}(0)\right|}{\lambda} .
$$

This completes the induction procedure and proves the theorem.

The following are some commonly used examples of loss functions (e.g. [4], [18], [19], [12], [35]).

Corollary 5: Let $\lambda>0$ and $\left\{f_{t}\right\}$ be defined by (4). Then the learning sequence $\left\{f_{t}\right\}$ is uniformly bounded in $\mathcal{H}_{K}$ if each step size $\eta_{t}$ with $t \in \mathbb{N}$ satisfies

(1) $\eta_{t}\left(2 \kappa^{2}+\lambda\right) \leq 1$ for the least square loss $\phi(x)=(1-x)^{2}$;

(2) $\eta_{t}\left(4 \kappa^{2}+\lambda\right) \leq 1$ for the $q$-norm SVM loss $\phi(x)=$ $(1-x)_{+}^{q}$ with $1 \leq q \leq 2$;

(3) $\eta_{t}\left[\kappa^{2} q(q-1)\left(1+\kappa^{2} q / \lambda\right)^{q-2}+\lambda\right] \leq 1$ for the $q$-norm SVM loss with $q>2$;

(4) $\eta_{t}\left[\kappa^{2} e^{\kappa^{2} / \lambda}+\lambda\right] \leq 1$ for the exponential loss $\phi(x)=$ $e^{-x}$.

Proof: Our conclusion follows from Theorem 4 and the expressions for the local Lipschitz constant $M(\lambda)$ derived separately.

(1) Note the least square loss $\phi$ is twice continuously differentiable and $\phi^{\prime \prime} \equiv 2$. Then we have $M(\lambda) \equiv 2$.

(2) When $1 \leq q \leq 2$, the $q$-norm SVM loss $\phi(x)=(1-x)_{+}^{q}$ has $\phi_{-}^{\prime}(x)=q(1-x)_{+}^{q-1}$. We have $M(\lambda) \leq 4$ for all $\lambda>0$ since

$$
\frac{\left|\phi_{-}^{\prime}(x)-\phi^{\prime}(0)\right|}{|x|} \leq \begin{cases}\left\|\phi^{\prime \prime}\right\|_{L^{\infty}[0,1 / 2]} \leq 4, & \text { if } x \in[0,1 / 2] \\ \left|\phi^{\prime}(0)\right| /|x| \leq 4, & \text { if } x>1 / 2, \\ \left\|\phi^{\prime \prime}\right\|_{L^{\infty}[-\infty, 0]} \leq 2, & \text { if } x<0 .\end{cases}
$$

(3) When $q>2$, we have $\phi^{\prime}(0)=-q$ and $\phi_{-}^{\prime \prime}(x)=q(q-$ $1)(1-x)_{+}^{q-2}$. So we find that $M(\lambda) \leq q(q-1)\left(1+\kappa^{2} q / \lambda\right)^{q-2}$.

(4) For the exponential loss, we have $\phi^{\prime}(x)=$ $-e^{-x}$ and $\phi^{\prime \prime}(x)=e^{-x}$. Then we have $M(\lambda) \leq$ $\left\|\phi^{\prime \prime}\right\|_{L^{\infty}\left[-\kappa^{2}\left|\phi^{\prime}(0)\right| / \lambda, \kappa^{2}\left|\phi^{\prime}(0)\right| / \lambda\right]}=e^{\kappa^{2} / \lambda}$.

\section{EXCESS GENERALIZATION ERROR AND RKHS METRIC}

In this section, we prove Theorem 2 , a relation between $\| f-$ $f_{\lambda} \|_{K}$ and $\left\{\mathcal{E}(f)+\frac{\lambda}{2}\|f\|_{K}^{2}\right\}-\left\{\mathcal{E}\left(f_{\lambda}\right)+\frac{\lambda}{2}\left\|f_{\lambda}\right\|_{K}^{2}\right\}$. This relation is very important for the proof of the general convergence result, Theorem 1, as well as for the error analysis done in the next section.

Lemma 1: Assume $\phi$ is differentiable. Then $f_{\lambda}$ satisfies

$\int_{Z} \phi^{\prime}\left(y f_{\lambda}(x)\right) y\left\{f(x)-f_{\lambda}(x)\right\} d \rho+\lambda\left\langle f_{\lambda}, f-f_{\lambda}\right\rangle_{K}=0$

for any $f \in \mathcal{H}_{K}$.

Proof: Since $f_{\lambda}$ is a minimizer of the regularized generalization error defined by (8), taking $f=0$ yields

$$
\mathcal{E}\left(f_{\lambda}\right)+\frac{\lambda}{2}\left\|f_{\lambda}\right\|_{K}^{2} \leq \mathcal{E}(0)=\phi(0) .
$$

Hence $\left\|f_{\lambda}\right\|_{\infty} \leq \kappa\left\|f_{\lambda}\right\|_{K} \leq \kappa \sqrt{2 \phi(0) / \lambda}$. Then for any $f \in$ $\mathcal{H}_{K}$ and $\theta>0$, we know that $\frac{1}{\theta}\left\{\left(\mathcal{E}\left(f_{\lambda}+\theta f\right)+\frac{\lambda}{2} \| f_{\lambda}+\right.\right.$ $\left.\left.\theta f \|_{K}^{2}\right)-\left(\mathcal{E}\left(f_{\lambda}\right)+\frac{\lambda}{2}\left\|f_{\lambda}\right\|_{K}^{2}\right)\right\}$ is nonnegative and equals

$$
\begin{aligned}
& \int_{Z} \frac{1}{\theta y f(x)}\left\{\phi\left(y f_{\lambda}(x)+\theta y f(x)\right)-\phi\left(y f_{\lambda}(x)\right)\right\} y f(x) d \rho \\
& +\lambda\left\langle f_{\lambda}, f\right\rangle_{K}+\frac{\lambda}{2} \theta\|f\|_{K}^{2} .
\end{aligned}
$$

Letting $\theta \rightarrow 0_{+}$, by the Lebesgue Dominant Theorem, we see that

$$
\int_{Z} \phi^{\prime}\left(y f_{\lambda}(x)\right) y f(x) d \rho+\lambda\left\langle f_{\lambda}, f\right\rangle_{K} \geq 0 .
$$

But $f(x)=\left\langle K_{x}, f\right\rangle_{K}$. It follows that

$$
\left\langle\int_{Z} \phi^{\prime}\left(y f_{\lambda}(x)\right) y K_{x} d \rho+\lambda f_{\lambda}, f\right\rangle_{K} \geq 0 .
$$

This is true for every $f \in \mathcal{H}_{K}$, which implies

$$
\int_{Z} \phi^{\prime}\left(y f_{\lambda}(x)\right) y K_{x} d \rho+\lambda f_{\lambda}=0 .
$$

Taking inner products with $f-f_{\lambda}$ in $\mathcal{H}_{K}$ proves the lemma.

We first prove Theorem 2 for differentiable loss functions.

Lemma 2: Let $\lambda>0$ and $\phi$ be a differentiable convex loss function. Then for any $f \in \mathcal{H}_{K}$ there holds

$\frac{\lambda}{2}\left\|f-f_{\lambda}\right\|_{K}^{2} \leq\left\{\mathcal{E}(f)+\frac{\lambda}{2}\|f\|_{K}^{2}\right\}-\left\{\mathcal{E}\left(f_{\lambda}\right)+\frac{\lambda}{2}\left\|f_{\lambda}\right\|_{K}^{2}\right\}$.

Proof: Let $f \in \mathcal{H}_{K}$. Define a univariate function $G=$ $G_{f}$ by

$G(\theta)=\mathcal{E}\left(f_{\lambda}+\theta\left(f-f_{\lambda}\right)\right)+\frac{\lambda}{2}\left\|f_{\lambda}+\theta\left(f-f_{\lambda}\right)\right\|_{K}^{2}, \quad \theta \in \mathbb{R}$.

We have

$$
G(1)=\mathcal{E}(f)+\frac{\lambda}{2}\|f\|_{K}^{2}, \quad G(0)=\mathcal{E}\left(f_{\lambda}\right)+\frac{\lambda}{2}\left\|f_{\lambda}\right\|_{K}^{2} .
$$

Since $\phi$ is differentiable, as a function of $\theta, G$ is differentiable. In fact, if we denote $f_{\theta}=f_{\lambda}+\theta\left(f-f_{\lambda}\right)$, then $G^{\prime}(\theta)=$ $\lim _{\Delta \theta \rightarrow 0} \frac{1}{\Delta \theta}\{G(\theta+\Delta \theta)-G(\theta)\}$ equals

$$
\begin{aligned}
& \lambda\left\langle f_{\theta}, f-f_{\lambda}\right\rangle_{K}+\lim _{\Delta \theta \rightarrow 0} \int_{Z} \frac{y\left(f(x)-f_{\lambda}(x)\right)}{\Delta \theta y\left(f(x)-f_{\lambda}(x)\right)} \\
& \left\{\phi\left(y f_{\theta}(x)+\Delta \theta y\left(f(x)-f_{\lambda}(x)\right)\right)-\phi\left(y f_{\theta}(x)\right)\right\} d \rho .
\end{aligned}
$$


The Lebesgue Dominant Theorem ensures that

$G^{\prime}(\theta)=\lambda\left\langle f_{\theta}, f-f_{\lambda}\right\rangle_{K}+\int_{Z} \phi^{\prime}\left(y f_{\theta}(x)\right) y\left(f(x)-f_{\lambda}(x)\right) d \rho$.

This in connection with Lemma 1 tells us that $G^{\prime}(\theta)$ equals

$$
\begin{aligned}
& \lambda \theta\left\|f-f_{\lambda}\right\|_{K}^{2}+\int_{Z}\left[\phi^{\prime}\left(y f_{\lambda}(x)+\theta y\left(f(x)-f_{\lambda}(x)\right)\right)\right. \\
& \left.-\phi^{\prime}\left(y f_{\lambda}(x)\right)\right] y\left(f(x)-f_{\lambda}(x)\right) d \rho .
\end{aligned}
$$

Since $\phi$ is convex in $\mathbb{R}$, it satisfies

$$
\left(\phi^{\prime}\left(x_{1}\right)-\phi^{\prime}\left(x_{2}\right)\right)\left(x_{1}-x_{2}\right) \geq 0 \quad \forall x_{1}, x_{2} \in \mathbb{R} .
$$

Using this for $x_{1}=y f_{\lambda}(x)+\theta y\left(f(x)-f_{\lambda}(x)\right)$ and $x_{2}=$ $y f_{\lambda}(x)$, we see from (37) that for $\theta \in(0,1)$,

$$
G^{\prime}(\theta) \geq \lambda \theta\left\|f-f_{\lambda}\right\|_{K}^{2} .
$$

Therefore

$$
\begin{aligned}
G(1)-G(0) & =\left\{\mathcal{E}(f)+\frac{\lambda}{2}\|f\|_{K}^{2}\right\}-\left\{\mathcal{E}\left(f_{\lambda}\right)+\frac{\lambda}{2}\left\|f_{\lambda}\right\|_{K}^{2}\right\} \\
& =\int_{0}^{1} G^{\prime}(\theta) d \theta \geq \frac{\lambda}{2}\left\|f-f_{\lambda}\right\|_{K}^{2} .
\end{aligned}
$$

This proves the desired result.

The intermediate step (37) in the above proof yields an interesting result for the least-square loss which may be useful in analyzing the Tikhonov regularization scheme (2).

Corollary 6: Let $\phi(x)=(1-x)^{2}$ be the least square loss. For any $f \in \mathcal{H}_{K}$, there holds

$$
\begin{aligned}
& \left\|f-f_{\lambda}\right\|_{L_{\rho_{X}}^{2}}^{2}+\frac{\lambda}{2}\left\|f-f_{\lambda}\right\|_{K}^{2} \\
& =\left\{\mathcal{E}(f)+\frac{\lambda}{2}\|f\|_{K}^{2}\right\}-\left\{\mathcal{E}\left(f_{\lambda}\right)+\frac{\lambda}{2}\left\|f_{\lambda}\right\|_{K}^{2}\right\} .
\end{aligned}
$$

Proof: It follows directly from (37) since $\phi^{\prime}(x)=2 x-2$ implies $G^{\prime}(\theta)=2 \theta\left\|f-f_{\lambda}\right\|_{L^{2}}^{2}+\lambda \theta\left\|f-f_{\lambda}\right\|_{K}^{2}$.

If $\phi$ is not differentiable like the hinge loss, we approximate it by $\phi_{\varepsilon}$ which is convex, differentiable and defined for $0<$ $\varepsilon \leq 1$ as

$$
\phi_{\varepsilon}(x):=\int_{0}^{1} \phi(x-\varepsilon \theta) d \theta=\frac{1}{\varepsilon} \int_{x-\varepsilon}^{x} \phi(u) d u .
$$

The approximation is valid: $\left|\phi_{\varepsilon}(x)-\phi(x)\right|=\mid \int_{0}^{1} \phi(x-\varepsilon u)-$ $\phi(x) d u \mid \leq\left\|\phi_{-}^{\prime}\right\|_{L^{\infty}[x-\varepsilon, x]} \varepsilon$. Hence for any $R>0$, there holds

$$
\left\|\phi_{\varepsilon}-\phi\right\|_{C[-R, R]}=O(\varepsilon) \quad \text { as } \varepsilon \rightarrow 0_{+} .
$$

Now we can prove Theorem 2 for a general loss function.

Proof of Theorem 2: We define, for any $0 \leq \varepsilon \leq 1$, $\mathcal{E}^{(\varepsilon)}(f)=\int_{Z} \phi_{\varepsilon}(y f(x)) d \rho$ and

$$
f_{\lambda}^{(\varepsilon)}=\arg \inf _{f \in \mathcal{H}_{K}}\left\{\mathcal{E}^{(\varepsilon)}(f)+\frac{\lambda}{2}\|f\|_{K}^{2}\right\} .
$$

For $\varepsilon=0$, we have used the conventional notation $\mathcal{E}^{(0)}(f)=$ $\mathcal{E}(f)$ and $f_{\lambda}^{(0)}=f_{\lambda}$.

Since $f_{\lambda}^{(\varepsilon)}$ is the minimizer of (39), by taking $f=0$ we get

$$
\mathcal{E}^{(\varepsilon)}\left(f_{\lambda}^{(\varepsilon)}\right)+\frac{\lambda}{2}\left\|f_{\lambda}^{(\varepsilon)}\right\|_{K}^{2} \leq \mathcal{E}^{(\varepsilon)}(0)=\phi_{\varepsilon}(0) \leq\|\phi\|_{C[-1,0]}<\infty
$$

which implies

$$
\left\|f_{\lambda}^{(\varepsilon)}\right\|_{K} \leq \sqrt{2\|\phi\|_{C[-1,0]} / \lambda} \quad \text { for any } \quad 0 \leq \varepsilon \leq 1
$$

Since any closed ball $B_{R}=\left\{f \in \mathcal{H}_{K}:\|f\|_{K} \leq R\right\}$ of the Hilbert space $\mathcal{H}_{K}$ is weakly compact, the estimate (40) tells us that there exists a sequence $\left\{\varepsilon_{j}>0\right\}_{j=1}^{\infty}$ such that $\lim _{j \rightarrow \infty} \varepsilon_{j}=0$ and $f_{\lambda}^{\left(\varepsilon_{j}\right)}$ converges to some $f_{\lambda}^{*} \in \mathcal{H}_{K}$ weakly. That is,

$$
\lim _{j \rightarrow \infty}\left\langle f_{\lambda}^{\left(\varepsilon_{j}\right)}, f\right\rangle_{K}=\left\langle f_{\lambda}^{*}, f\right\rangle_{K}, \quad \forall f \in \mathcal{H}_{K}
$$

In particular, $\left\|f_{\lambda}^{*}\right\|_{K}^{2}=\left\langle f_{\lambda}^{*}, f_{\lambda}^{*}\right\rangle_{K}=\lim _{j \rightarrow \infty}\left\langle f_{\lambda}^{\left(\varepsilon_{j}\right)}, f_{\lambda}^{*}\right\rangle_{K} \leq$ $\left\|f_{\lambda}^{*}\right\|_{K} \underline{\lim }_{j \rightarrow \infty}\left\|f_{\lambda}^{\left(\varepsilon_{j}\right)}\right\|_{K}$ and

$$
\left\|f_{\lambda}^{*}\right\|_{K} \leq \underline{\lim }_{j \rightarrow \infty}\left\|f_{\lambda}^{\left(\varepsilon_{j}\right)}\right\|_{K} \leq \sqrt{2\|\phi\|_{C[-1,0]} / \lambda}
$$

Let $f=K_{x}$ in (41). Then the reproducing property (1) yields

$$
\lim _{j \rightarrow \infty} f_{\lambda}^{\left(\varepsilon_{j}\right)}(x)=\lim _{j \rightarrow \infty}\left\langle f_{\lambda}^{\left(\varepsilon_{j}\right)}, K_{x}\right\rangle_{K}=\left\langle f_{\lambda}^{*}, K_{x}\right\rangle_{K}=f_{\lambda}^{*}(x) .
$$

This in connection with the continuity of $\phi$ and the Lebesgue Dominant Theorem gives $\mathcal{E}\left(f_{\lambda}^{*}\right)=$ $\lim _{j \rightarrow \infty} \int_{Z} \phi\left(y f_{\lambda}^{\left(\varepsilon_{j}\right)}(x)\right) d \rho$. The uniform bound (40) of $\left\|f_{\lambda}^{\left(\varepsilon_{j}\right)}\right\|_{K}$ in connection with the uniform convergence (38) of $\phi_{\varepsilon}$ to $\phi$ ensures that

$$
\begin{aligned}
& \underline{\lim }_{j \rightarrow \infty} \mathcal{E}^{\left(\varepsilon_{j}\right)}\left(f_{\lambda}^{\left(\varepsilon_{j}\right)}\right)=\lim _{j \rightarrow \infty} \int_{Z} \phi^{\left(\varepsilon_{j}\right)}\left(y f_{\lambda}^{\left(\varepsilon_{j}\right)}(x)\right) d \rho \\
& =\lim _{j \rightarrow \infty} \int_{Z} \phi\left(y f_{\lambda}^{\left(\varepsilon_{j}\right)}(x)\right) d \rho=\mathcal{E}\left(f_{\lambda}^{*}\right) .
\end{aligned}
$$

Therefore, by (42), we have

$$
\mathcal{E}\left(f_{\lambda}^{*}\right)+\frac{\lambda}{2}\left\|f_{\lambda}^{*}\right\|_{K}^{2} \leq \underline{\lim }_{j \rightarrow \infty}\left\{\mathcal{E}^{\left(\varepsilon_{j}\right)}\left(f_{\lambda}^{\left(\varepsilon_{j}\right)}\right)+\frac{\lambda}{2}\left\|f_{\lambda}^{\left(\varepsilon_{j}\right)}\right\|_{K}^{2}\right\} .
$$

Taking $f=f_{\lambda}$ in (39), we know that

$$
\begin{aligned}
& \underline{\lim }_{j \rightarrow \infty}\left\{\mathcal{E}^{\left(\varepsilon_{j}\right)}\left(f_{\lambda}^{\left(\varepsilon_{j}\right)}\right)+\frac{\lambda}{2}\left\|f_{\lambda}^{\left(\varepsilon_{j}\right)}\right\|_{K}^{2}\right\} \\
& \leq \underline{\lim }_{j \rightarrow \infty}\left\{\mathcal{E}^{\left(\varepsilon_{j}\right)}\left(f_{\lambda}\right)+\frac{\lambda}{2}\left\|f_{\lambda}\right\|_{K}^{2}\right\}=\mathcal{E}\left(f_{\lambda}\right)+\frac{\lambda}{2}\left\|f_{\lambda}\right\|_{K}^{2}
\end{aligned}
$$

which means

$$
\mathcal{E}\left(f_{\lambda}^{*}\right)+\frac{\lambda}{2}\left\|f_{\lambda}^{*}\right\|_{K}^{2} \leq \mathcal{E}\left(f_{\lambda}\right)+\frac{\lambda}{2}\left\|f_{\lambda}\right\|_{K}^{2} .
$$

It tells us that $f_{\lambda}^{*}$ is also a minimizer of $\mathcal{E}(f)+\frac{\lambda}{2}\|f\|_{K}^{2}$. The strict convexity of the functional $\mathcal{E}(f)+\frac{\lambda}{2}\|f\|_{K}^{2}$ on $\mathcal{H}_{K}$ verifies the uniqueness of $f_{\lambda}$ which leads to $f_{\lambda}^{*}=f_{\lambda}$. That is, (41) and (42) hold with $f_{\lambda}^{*}$ replaced by $f_{\lambda}$.

Apply Lemma 2 to the modified loss function $\phi_{\varepsilon_{j}}$. We have

$$
\begin{aligned}
& \frac{\lambda}{2}\left\|f-f_{\lambda}^{\left(\varepsilon_{j}\right)}\right\|_{K}^{2} \leq\left\{\mathcal{E}^{\left(\varepsilon_{j}\right)}(f)+\frac{\lambda}{2}\|f\|_{K}^{2}\right\} \\
& -\left\{\mathcal{E}^{\left(\varepsilon_{j}\right)}\left(f_{\lambda}^{\left(\varepsilon_{j}\right)}\right)+\frac{\lambda}{2}\left\|f_{\lambda}^{\left(\varepsilon_{j}\right)}\right\|_{K}^{2}\right\} .
\end{aligned}
$$


Applying (41) to $f-f_{\lambda}$, we know that

$$
\begin{aligned}
& \left\|f-f_{\lambda}\right\|_{K}^{2}=\left\langle f, f-f_{\lambda}\right\rangle_{K}-\lim _{j \rightarrow \infty}\left\langle f_{\lambda}^{\left(\varepsilon_{j}\right)}, f-f_{\lambda}\right\rangle_{K} \\
& =\lim _{j \rightarrow \infty}\left\langle f-f_{\lambda}^{\left(\varepsilon_{j}\right)}, f-f_{\lambda}\right\rangle_{K}
\end{aligned}
$$

which can be bounded by $\underline{\lim }_{j \rightarrow \infty}\left\|f-f_{\lambda}^{\left(\varepsilon_{j}\right)}\right\|_{K}\left\|f-f_{\lambda}\right\|_{K}$. Hence

$$
\frac{\lambda}{2}\left\|f-f_{\lambda}\right\|_{K} \leq \frac{\lambda}{2} \underline{\lim }_{j \rightarrow \infty}\left\|f-f_{\lambda}^{\left(\varepsilon_{j}\right)}\right\|_{K}
$$

This in connection with (45), (42), and (44) implies that $\frac{\lambda}{2} \| f-$ $f_{\lambda} \|_{K}^{2}$ is bounded by

$$
\left\{\lim _{j \rightarrow \infty} \mathcal{E}^{\left(\varepsilon_{j}\right)}(f)+\frac{\lambda}{2}\|f\|_{K}^{2}\right\}-\left\{\mathcal{E}\left(f_{\lambda}\right)+\frac{\lambda}{2}\left\|f_{\lambda}\right\|_{K}^{2}\right\} .
$$

Since $\lim _{j \rightarrow \infty} \mathcal{E}^{\left(\varepsilon_{j}\right)}(f)=\mathcal{E}(f)$, the conclusion of Theorem 2 is proved.

\section{General Convergence Results}

In this section we prove our first main result, Theorem 1. The essential estimate in the proof will also be used in the next section to give convergence rates. Note that the uniform boundedness of $\left\|f_{t}\right\|_{K}$ by $B$ implies $\| \phi_{-}^{\prime}\left(y_{t} f_{t}\left(x_{t}\right)\right) y_{t} K_{x_{t}}+$ $\lambda f_{t}\left\|_{K} \leq \kappa\right\| \phi_{-}^{\prime} \|_{L^{\infty}[-\kappa B, \kappa B]}+\lambda B$.

For simplicity, denote $\Pi_{j=T+1}^{T}\left(1-\eta_{j} \lambda\right)=1$ and $\sum_{j=T+1}^{T} \eta_{j} \lambda=0$.

Lemma 3: Assume that for some $t_{0} \in \mathbb{N}$ and $\widetilde{C}_{\lambda}>0$, there holds

$$
\eta_{t} \lambda<1, \quad \mathbb{E}_{z_{1}, \ldots, z_{t}}\left(\left\|\phi_{-}^{\prime}\left(y_{t} f_{t}\left(x_{t}\right)\right) y_{t} K_{x_{t}}+\lambda f_{t}\right\|_{K}^{2}\right) \leq \widetilde{C}_{\lambda}
$$

for any $t \geq t_{0}$. Then for $T>t_{0}$,

$$
\begin{aligned}
& \mathbb{E}_{z_{1}, \ldots, z_{T}}\left(\left\|f_{T+1}-f_{\lambda}\right\|_{K}^{2}\right) \leq \widetilde{C}_{\lambda} \sum_{t=t_{0}}^{T} \eta_{t}^{2} \prod_{j=t+1}^{T}\left(1-\eta_{j} \lambda\right) \\
& +\prod_{t=t_{0}}^{T}\left(1-\eta_{t} \lambda\right) \mathbb{E}_{z_{1}, \ldots, z_{t_{0}-1}}\left(\left\|f_{t_{0}}-f_{\lambda}\right\|_{K}^{2}\right)
\end{aligned}
$$

which can be further controlled by

$$
\begin{aligned}
& \exp \left\{-\sum_{t=t_{0}}^{T} \eta_{t} \lambda\right\} \mathbb{E}_{z_{1}, \ldots, z_{t_{0}-1}}\left(\left\|f_{t_{0}}-f_{\lambda}\right\|_{K}^{2}\right) \\
& +\widetilde{C}_{\lambda} \sum_{t=t_{0}}^{T} \eta_{t}^{2} \exp \left\{-\sum_{j=t+1}^{T} \eta_{j} \lambda\right\} .
\end{aligned}
$$

Proof: Recall that $f_{t+1}=f_{t}-\eta_{t} f_{t}^{\lambda}$ where $f_{t}^{\lambda}=$ $\phi_{-}^{\prime}\left(y_{t} f_{t}\left(x_{t}\right)\right) y_{t} K_{x_{t}}+\lambda f_{t}$. Then

$\left\|f_{t+1}-f_{\lambda}\right\|_{K}^{2}=\left\|f_{t}-f_{\lambda}\right\|_{K}^{2}+\eta_{t}^{2}\left\|f_{t}^{\lambda}\right\|_{K}^{2}+2 \eta_{t}\left\langle f_{t}^{\lambda}, f_{\lambda}-f_{t}\right\rangle_{K}$.

By the reproducing property (1), part of the last term of (49) $\left\langle\phi_{-}^{\prime}\left(y_{t} f_{t}\left(x_{t}\right)\right) y_{t} K_{x_{t}}, f_{\lambda}-f_{t}\right\rangle_{K}$ equals

$$
\phi_{-}^{\prime}\left(y_{t} f_{t}\left(x_{t}\right)\right) y_{t}\left(f_{\lambda}\left(x_{t}\right)-f_{t}\left(x_{t}\right)\right) .
$$

Since $\phi$ is a convex function on $\mathbb{R}$, we know that

$$
\phi_{-}^{\prime}(a)(b-a) \leq \phi(b)-\phi(a), \quad \forall a, b \in \mathbb{R} .
$$

Applying this relation to $a=y_{t} f_{t}\left(x_{t}\right)$ and $b=y_{t} f_{\lambda}\left(x_{t}\right)$ together with (50) yields

$\left\langle\phi_{-}^{\prime}\left(y_{t} f_{t}\left(x_{t}\right)\right) y_{t} K_{x_{t}}, f_{\lambda}-f_{t}\right\rangle_{K} \leq \phi\left(y_{t} f_{\lambda}\left(x_{t}\right)\right)-\phi\left(y_{t} f_{t}\left(x_{t}\right)\right)$.

The Schwarz inequality $\left\langle f_{t}, f_{\lambda}\right\rangle_{K} \leq\left\|f_{t}\right\|_{K}\left\|f_{\lambda}\right\|_{K} \leq$ $\frac{1}{2}\left(\left\|f_{\lambda}\right\|_{K}^{2}+\left\|f_{t}\right\|_{K}^{2}\right)$ implies

$$
\lambda\left\langle f_{t}, f_{\lambda}-f_{t}\right\rangle_{K} \leq \frac{\lambda}{2}\left\|f_{\lambda}\right\|_{K}^{2}-\frac{\lambda}{2}\left\|f_{t}\right\|_{K}^{2} .
$$

Putting this and (51) into the middle term of (49), we know that $\left\langle f_{t}^{\lambda}, f_{\lambda}-f_{t}\right\rangle_{K}$ is bounded by

$$
\left[\phi\left(y_{t} f_{\lambda}\left(x_{t}\right)\right)+\frac{\lambda}{2}\left\|f_{\lambda}\right\|_{K}^{2}\right]-\left[\phi\left(y_{t} f_{t}\left(x_{t}\right)\right)+\frac{\lambda}{2}\left\|f_{t}\right\|_{K}^{2}\right] .
$$

Since $f_{t}$ depends on $\left\{z_{1}, \cdots, z_{t-1}\right\}$ but not on $z_{t}$, it follows that $\mathbb{E}_{z_{1}, z_{2}, \cdots, z_{t}}\left(\left\langle f_{t}^{\lambda}, f_{\lambda}-f_{t}\right\rangle_{K}\right)$ can be bounded by

$$
\begin{aligned}
& \mathbb{E}_{z_{1}, \cdots, z_{t-1}}\left(\mathbb { E } _ { z _ { t } } \left(\left[\phi\left(y_{t} f_{\lambda}\left(x_{t}\right)\right)+\frac{\lambda}{2}\left\|f_{\lambda}\right\|_{K}^{2}\right]\right.\right. \\
& \left.\left.-\left[\phi\left(y_{t} f_{t}\left(x_{t}\right)\right)+\frac{\lambda}{2}\left\|f_{t}\right\|_{K}^{2}\right]\right)\right) \\
& =\mathbb{E}_{z_{1}, \cdots, z_{t-1}}\left(\left[\mathcal{E}\left(f_{\lambda}\right)+\frac{\lambda}{2}\left\|f_{\lambda}\right\|_{K}^{2}\right]\right. \\
& \left.-\left[\mathcal{E}\left(f_{t}\right)+\frac{\lambda}{2}\left\|f_{t}\right\|_{K}^{2}\right]\right) .
\end{aligned}
$$

This in connection with (46) and (49) gives

$$
\begin{aligned}
& \mathbb{E}_{z_{1}, \cdots, z_{t}}\left(\left\|f_{t+1}-f_{\lambda}\right\|_{K}^{2}\right) \leq \mathbb{E}_{z_{1}, \cdots, z_{t-1}}\left(\left\|f_{t}-f_{\lambda}\right\|_{K}^{2}\right) \\
& +\widetilde{C}_{\lambda} \eta_{t}^{2}+2 \eta_{t} \mathbb{E}_{z_{1}, \cdots, z_{t-1}}\left(\left[\mathcal{E}\left(f_{\lambda}\right)+\frac{\lambda}{2}\left\|f_{\lambda}\right\|_{K}^{2}\right]\right. \\
& \left.-\left[\mathcal{E}\left(f_{t}\right)+\frac{\lambda}{2}\left\|f_{t}\right\|_{K}^{2}\right]\right) .
\end{aligned}
$$

By Theorem 2, this implies that $\mathbb{E}_{z_{1}, \cdots, z_{t}}\left(\left\|f_{t+1}-f_{\lambda}\right\|_{K}^{2}\right)$ is bounded by

$$
\left(1-\eta_{t} \lambda\right) \mathbb{E}_{z_{1}, \cdots, z_{t-1}}\left(\left\|f_{t}-f_{\lambda}\right\|_{K}^{2}\right)+\widetilde{C}_{\lambda} \eta_{t}^{2} .
$$

Applying this relation iteratively for $t=T, T-1, \ldots, t_{0}$, we see that $\mathbb{E}_{z_{1}, \ldots, z_{T}}\left(\left\|f_{T+1}-f_{\lambda}\right\|_{K}^{2}\right)$ is bounded by

$$
\begin{aligned}
& \left(1-\eta_{T} \lambda\right)\left(1-\eta_{T-1} \lambda\right) \mathbb{E}_{z_{1}, \cdots, z_{T-2}}\left(\left\|f_{T-1}-f_{\lambda}\right\|_{K}^{2}\right) \\
& +\widetilde{C}_{\lambda} \eta_{T}^{2}+\left(1-\eta_{T} \lambda\right) \widetilde{C}_{\lambda} \eta_{T-1}^{2} \leq \ldots \\
& \leq \prod_{t=t_{0}}^{T}\left(1-\eta_{t} \lambda\right) \mathbb{E}_{z_{1}, \ldots, z_{t_{0}-1}}\left(\left\|f_{t_{0}}-f_{\lambda}\right\|_{K}^{2}\right) \\
& +\widetilde{C}_{\lambda} \sum_{t=t_{0}}^{T} \eta_{t}^{2} \prod_{j=t+1}^{T}\left(1-\eta_{j} \lambda\right) .
\end{aligned}
$$

This proves the first statement.

The second statement follows from the inequality $1-u \leq$ $e^{-u}$ for any $u \geq 0$.

We are in a position to prove Theorem 1 stated in the introduction. For this purpose we use (47) while the bound (48) will be used to derive explicit learning rates in the next section.

Proof of Theorem 1: By (6), there exists an integer $t_{0}$ such that $\eta_{t} \lambda \leq 1 / 2$ for all $t \geq t_{0}$. Since $\left\{f_{t}\right\}$ is uniformly bounded in $\mathcal{H}_{K}$, (46) is true for some constant $\widetilde{C}_{\lambda}$. Applying Lemma 3 , it is sufficient to estimate the right side of (47). According to 
the assumption (6) on the step size, we have $\prod_{t=t_{0}}^{T}\left(1-\eta_{t} \lambda\right) \leq$ $\exp \left\{-\sum_{t=t_{0}}^{T} \eta_{t} \lambda\right\} \rightarrow 0$ as $T \rightarrow \infty$. So for any $\varepsilon>0$ there exists some $T_{1} \in \mathbb{N}$ such that the second term of (47) is bounded by $\varepsilon$ whenever $T \geq T_{1}$.

To deal with the first term, we use the assumption $\lim _{t \rightarrow \infty} \eta_{t}=0$ and know that there exists some $t(\varepsilon)$ such that $\eta_{t} \leq \lambda \varepsilon$ for every $t \geq t(\varepsilon)$. Write $\sum_{t=t_{0}}^{T} \eta_{t}^{2} \Pi_{j=t+1}^{T}\left(1-\eta_{j} \lambda\right)$ as

$$
\sum_{t=t_{0}}^{t(\varepsilon)} \eta_{t}^{2} \Pi_{j=t+1}^{T}\left(1-\eta_{j} \lambda\right)+\sum_{t=t(\varepsilon)+1}^{T} \eta_{t}^{2} \Pi_{j=t+1}^{T}\left(1-\eta_{j} \lambda\right) .
$$

Since $t(\varepsilon)$ is fixed, we can find some $T_{2} \in \mathbb{N}$ such that for each $T \geq T_{2}$, there holds $\sum_{j=t(\varepsilon)+1}^{T} \eta_{j} \geq \sum_{j=t(\varepsilon)+1}^{T_{2}} \eta_{j} \geq$ $\frac{1}{\lambda} \log \frac{t(\varepsilon)}{4 \lambda^{2} \varepsilon}$. It follows that for each $t_{0} \leq t \leq t(\varepsilon)$, there holds $\Pi_{j=t+1}^{T}\left(1-\eta_{j} \lambda\right) \leq \exp \left\{-\sum_{j=t+1}^{T} \eta_{j} \lambda\right\} \leq$ $\exp \left\{-\sum_{j=t(\varepsilon)+1}^{T} \eta_{j} \lambda\right\} \leq \frac{4 \lambda^{2} \varepsilon}{t(\varepsilon)}$. This in connection with the bound $\eta_{t} \lambda \leq 1 / 2$ for each $t \geq t_{0}$ tells us that the first term of (55) is bounded as

$$
\sum_{t=t_{0}}^{t(\varepsilon)} \eta_{t}^{2} \Pi_{j=t+1}^{T}\left(1-\eta_{j} \lambda\right) \leq \frac{4 \lambda^{2} \varepsilon}{t(\varepsilon)} \sum_{t=t_{0}}^{t(\varepsilon)} \eta_{t}^{2} \leq \varepsilon .
$$

The second term of (55) is dominated by $\lambda \varepsilon \sum_{t=t(\varepsilon)+1}^{T-1} \eta_{t} \Pi_{j=t+1}^{T}\left(1-\eta_{j} \lambda\right)$. But $\eta_{t} \lambda=1-\left(1-\eta_{t} \lambda\right)$. Then

$$
\begin{aligned}
& \lambda \sum_{t=t(\varepsilon)+1}^{T} \eta_{t} \Pi_{j=t+1}^{T}\left(1-\eta_{j} \lambda\right) \\
= & \sum_{t=t(\varepsilon)+1}^{T}\left[\Pi_{j=t+1}^{T}\left(1-\eta_{j} \lambda\right)-\Pi_{j=t}^{T}\left(1-\eta_{j} \lambda\right)\right] \\
= & {\left[1-\Pi_{j=t(\varepsilon)+1}^{T}\left(1-\eta_{j} \lambda\right)\right] \leq 1 . }
\end{aligned}
$$

Therefore, when $T \geq \max \left\{T_{1}, T_{2}\right\}$, by Lemma 3, we have $\mathbb{E}\left(\left\|f_{T+1}-f_{\lambda}\right\|_{K}^{2}\right) \leq\left(1+2 \widetilde{C}_{\lambda}\right) \varepsilon$. This proves Theorem 1 .

\section{Convergence Rates}

Now we can derive convergence rates for the error $\| f_{T+1}-$ $f_{\lambda} \|_{K}$. The step size here is often of the form $\eta_{t}=\frac{1}{\mu(\lambda) t^{\theta}}$ for some $\theta \in(0,1]$ and $\mu(\lambda)>0$. So to apply Lemma 3 for getting error bounds, we need to estimate the summations in (48) and lead to the following lemmas.

Lemma 4: For any $t<T$ and $0<\theta \leq 1$, there holds

$$
\sum_{j=t+1}^{T} j^{-\theta} \geq \begin{cases}\frac{1}{1-\theta}\left[(T+1)^{1-\theta}-(t+1)^{1-\theta}\right] & \text { if } \theta<1, \\ \log (T+1)-\log (t+1) & \text { if } \theta=1 .\end{cases}
$$

The proof follows from the simple inequality $\sum_{j=t+1}^{T} j^{-\theta} \geq \int_{t+1}^{T+1} u^{-\theta} d u$.

The next lemma in a modified form was given in [22] for $1 / 2<\theta<1$.

Lemma 5: Let $0<\nu \leq 1$ and $0<\theta \leq 1$. Then $\sum_{t=1}^{T-1} \frac{1}{t^{2 \theta}} \exp \left\{-\nu \sum_{j=t+1}^{T} j^{-\theta}\right\}$ is bounded by

$$
\begin{cases}\frac{18}{\nu T^{\theta}}+\frac{9 T^{1-\theta}}{(1-\theta) 2^{1-\theta}} \exp \left\{-\frac{\nu\left(1-2^{\theta-1}\right)}{1-\theta}(T+1)^{1-\theta}\right\}, & \theta<1, \\ \frac{8}{1-\nu}(T+1)^{-\nu}, & \theta=1 .\end{cases}
$$

Proof: Denote $I=\sum_{t=1}^{T-1} \frac{1}{t^{2 \theta}} \exp \left\{-\nu \sum_{j=t+1}^{T} j^{-\theta}\right\}$. For $\theta \in(0,1)$, we apply (56) in Lemma 4 and see that $I$ is bounded by

$\exp \left\{-\frac{\nu}{1-\theta}(T+1)^{1-\theta}\right\} \sum_{t=1}^{T-1} \frac{1}{t^{2 \theta}} \exp \left\{\frac{\nu}{1-\theta}(t+1)^{1-\theta}\right\}$.

For $x \in[t+1, t+2]$, we have $\frac{1}{t^{2 \theta}} \leq \frac{9}{x^{2 \theta}}$ and $\exp \left\{\frac{\nu}{1-\theta}(t+\right.$ $\left.1)^{1-\theta}\right\} \leq \exp \left\{\frac{\nu}{1-\theta} x^{1-\theta}\right\}$. Then $I$ is bounded by

$9 \exp \left\{-\frac{\nu}{1-\theta}(T+1)^{1-\theta}\right\} \int_{2}^{T+1} \frac{1}{x^{2 \theta}} \exp \left\{\frac{\nu}{1-\theta} x^{1-\theta}\right\} d x$.

Decompose the above integral in two parts. We have

$$
\begin{aligned}
& \int_{T / 2}^{T+1} \frac{1}{x^{2 \theta}} \exp \left\{\frac{\nu}{1-\theta} x^{1-\theta}\right\} d x \\
& \leq(T / 2)^{-\theta} \int_{T / 2}^{T+1} x^{-\theta} \exp \left\{\frac{\nu}{1-\theta} x^{1-\theta}\right\} d x \\
& \leq \frac{2^{\theta}}{\nu T^{\theta}} \exp \left\{\frac{\nu}{1-\theta}(T+1)^{1-\theta}\right\} .
\end{aligned}
$$

When $x \in[2, T / 2]$, we have $x^{-\theta} \leq 1$ and $\exp \left\{\frac{\nu}{1-\theta} x^{1-\theta}\right\} \leq$ $\exp \left\{\frac{\nu}{1-\theta}(T / 2)^{1-\theta}\right\}$. Also, $\int_{1}^{T / 2} x^{-\theta} d x \leq \frac{T^{1-\theta}}{(1-\theta) 2^{1-\theta}}$. Hence

$$
\begin{aligned}
& \int_{2}^{T / 2} \frac{1}{x^{2 \theta}} \exp \left\{\frac{\nu}{1-\theta} x^{1-\theta}\right\} d x \\
& \leq \exp \left\{\frac{\nu}{1-\theta}(T / 2)^{1-\theta}\right\} \frac{T^{1-\theta}}{(1-\theta) 2^{1-\theta}} .
\end{aligned}
$$

Combining (57), (58) and (59), we get

$$
I \leq \frac{18}{\nu T^{\theta}}+\frac{9 T^{1-\theta}}{(1-\theta) 2^{1-\theta}} \exp \left\{-\frac{\nu\left(1-2^{\theta-1}\right)}{1-\theta}(T+1)^{1-\theta}\right\} .
$$

For $\theta=1$, the inequality (56) implies $I$ is bounded by $\frac{1}{(T+1)^{\nu}} \sum_{t=1}^{T-1} \frac{(t+1)^{\nu}}{t^{2}}$. For $x \in[t, t+1]$, there holds $\frac{(t+1)^{\nu}}{t^{2}} \leq$ $2^{\nu+2} \frac{x^{\nu}}{x^{2}}$. Hence $I \leq \frac{8}{(T+1)^{\nu}} \int_{1}^{T} x^{\nu-2} d x \leq \frac{8}{(1-\nu)(T+1)^{\nu}}$, which proves the lemma.

We are in a position to state the convergence rates for the online algorithm (4). To this end, we need the following constant depending on $\lambda$ :

$$
\widetilde{C}_{\lambda}=4 \kappa^{2}\left\|\phi_{-}^{\prime}\right\|_{L^{\infty}\left[-\kappa^{2}\left|\phi^{\prime}(0)\right| / \lambda, \kappa^{2}\left|\phi^{\prime}(0)\right| / \lambda\right]}^{2} .
$$

Theorem 5: Assume that $\phi_{-}^{\prime}$ is locally Lipschitz at the origin. Choose the step sizes as $\eta_{t}=\frac{1}{\mu(\lambda) t^{\theta}}$ for some $\theta \in(0,1]$ and $\mu(\lambda) \geq M(\lambda) \kappa^{2}+\lambda$. Define $\left\{f_{t}\right\}$ by (4) and $\widetilde{C}_{\lambda}$ by (60).

1) For $0<\theta<1$,

$$
\begin{gathered}
\mathbb{E}\left(\left\|f_{T+1}-f_{\lambda}\right\|_{K}^{2}\right) \leq\left(\frac{2 \mathcal{D}(\lambda)}{\lambda}+\frac{9 \widetilde{C}_{\lambda} T^{1-\theta}}{(1-\theta) 2^{1-\theta}(\mu(\lambda))^{2}}\right) \\
\exp \left\{-\frac{\left(1-2^{\theta-1}\right) \lambda}{(1-\theta) \mu(\lambda)} T^{1-\theta}\right\}+\frac{19 \widetilde{C}_{\lambda}}{\mu(\lambda) \lambda T^{\theta}} .
\end{gathered}
$$

2) For $\theta=1, \mathbb{E}\left(\left\|f_{T+1}-f_{\lambda}\right\|_{K}^{2}\right)$ can be bounded by

$$
\left(\frac{2 \mathcal{D}(\lambda)}{\lambda}+\frac{9 \widetilde{C}_{\lambda}}{\mu(\lambda)(\mu(\lambda)-\lambda)}\right) T^{-\frac{\lambda}{\mu(\lambda)}} \text {. }
$$

Proof: The condition on the step size tells us that $\eta_{t}\left(M(\lambda) \kappa^{2}+\lambda\right) \leq 1$ for each $t$. By Theorem 4 , this yields 
$\left\|f_{t}\right\|_{K} \leq \kappa\left|\phi^{\prime}(0)\right| / \lambda$, and hence $\left|y_{t} f_{t}\left(x_{t}\right)\right| \leq\left\|f_{t}\right\|_{\infty} \leq$ $\kappa^{2}\left|\phi^{\prime}(0)\right| / \lambda$ for each $t$. Consequently, both $y_{t} f_{t}\left(x_{t}\right)$ and 0 lie in the interval $I_{\lambda}=\left[-\kappa^{2}\left|\phi^{\prime}(0)\right| / \lambda, \kappa^{2}\left|\phi^{\prime}(0)\right| / \lambda\right]$. It follows that $\left\|\phi_{-}^{\prime}\left(y_{t} f_{t}\left(x_{t}\right)\right) y_{t} K_{x_{t}}\right\|_{K} \leq \kappa\left\|\phi_{-}^{\prime}\right\|_{L^{\infty}\left(I_{\lambda}\right)}^{2}$ and $\left\|\lambda f_{t}\right\|_{K} \leq$ $\kappa\left|\phi^{\prime}(0)\right| \leq \kappa\left\|\phi_{-}^{\prime}\right\|_{L^{\infty}\left(I_{\lambda}\right)}^{2}$. Then (46) holds with $t_{0}=1$ and $\widetilde{C}_{\lambda}$. This in connection with (48) of Lemma 3 tells us that

$$
\mathbb{E}\left(\left\|f_{T+1}-f_{\lambda}\right\|_{K}^{2}\right) \leq I_{1}+I_{2},
$$

where

$$
\begin{aligned}
& I_{1}=\exp \left\{-\sum_{t=1}^{T} \frac{\lambda}{\mu(\lambda) t^{\theta}}\right\}\left\|f_{1}-f_{\lambda}\right\|_{K}^{2}, \\
& I_{2}=\frac{\widetilde{C}_{\lambda}}{(\mu(\lambda))^{2}} \sum_{t=1}^{T} \frac{1}{t^{2 \theta}} \exp \left\{-\frac{\lambda}{\mu(\lambda)} \sum_{j=t+1}^{T} j^{-\theta}\right\} .
\end{aligned}
$$

Since $f_{1}=0$, (24) gives $\left\|f_{1}-f_{\lambda}\right\|_{K}^{2} \leq 2 \mathcal{D}(\lambda) / \lambda$. By Lemma 4, we know that

$I_{1} \leq \begin{cases}\frac{2 \mathcal{D}(\lambda)}{\lambda} \exp \left\{-\frac{\left(1-2^{\theta-1}\right) \lambda}{(1-\theta) \mu(\lambda)}(T+1)^{1-\theta}\right\}, & \text { if } 0<\theta<1, \\ \frac{2 \mathcal{D}(\lambda)}{\lambda}(T+1)^{-\frac{\lambda}{\mu(\lambda)},} & \text { if } \theta=1 .\end{cases}$

By Lemma $5, I_{2}$ can be bounded by

$$
\left\{\begin{array}{cc}
\frac{\widetilde{C}_{\lambda}}{(\mu(\lambda))^{2}}\left\{\frac{9 T^{1-\theta}}{(1-\theta) 2^{1-\theta}} \exp \left\{-\frac{\left(1-2^{\theta-1}\right) \lambda}{(1-\theta) \mu(\lambda)}(T+1)^{1-\theta}\right\}\right. \\
\left.+\frac{18 \mu(\lambda) / \lambda}{T^{\theta}}+\frac{1}{T^{2 \theta}}\right\}, & \text { if } 0<\theta<1, \\
\frac{\widetilde{C}_{\lambda}}{(\mu(\lambda))^{2}}\left\{\frac{8}{1-\lambda / \mu(\lambda)}(T+1)^{-\frac{\lambda}{\mu(\lambda)}}+\frac{1}{T^{2}}\right\}, & \text { if } \theta=1 .
\end{array}\right.
$$

This proves Theorem 5 .

To apply Theorem 5 for deriving rates for the misclassification error, we need the constant $\widetilde{C}_{\lambda}$ and $\mu(\lambda)$. They depend on the loss function $\phi$ and play an essential role in getting learning rates. When $\phi_{-}^{\prime}$ satisfies the following increment condition with some $p \geq 0, c_{p}>0$ :

$$
\left|\phi_{-}^{\prime}(x)\right| \leq c_{p}|x|^{p}, \quad \forall|x| \geq 1,
$$

we can find $\widetilde{C}_{\lambda}$ and $\mu(\lambda)$ explicitly and then derive learning rates for the total error from Theorem 5. Denote $C_{\phi, \kappa}$ as a constant depending only on $\phi$ and $\kappa$ satisfying

$$
\begin{gathered}
C_{\phi, \kappa} \geq \max \left\{2 \kappa\left\|\phi_{-}^{\prime}\right\|_{L^{\infty}[-1,1]}^{1 / 2}, 2 \kappa^{1+p} \sqrt{c_{p}}\left|\phi^{\prime}(0)\right|^{p / 2},\right. \\
1+\kappa^{2}\left\|\left[\phi_{-}^{\prime}(x)-\phi^{\prime}(0)\right] / x\right\|_{L^{\infty}[-1,1]}, \\
\left.c_{p} \kappa^{2 p}\left|\phi^{\prime}(0)\right|^{p-1}+\kappa^{2}\left|\phi^{\prime}(0)\right|+1\right\} .
\end{gathered}
$$

Corollary 7: Assume that $\phi$ satisfies (63) and $\phi_{-}^{\prime}$ is locally Lipschitz at the origin. Let $0<\lambda \leq 1$. Choose the step sizes as $\eta_{t}=\frac{\lambda^{\max \{p-1,0\}}}{C_{\phi, \kappa} t^{\theta}}$ with some $0<\theta<1$ and $C_{\phi, \kappa}$ given by (64). Define $\left\{f_{t}\right\}$ by (4). Then

$$
\begin{gathered}
\mathbb{E}\left(\left\|f_{T+1}-f_{\lambda}\right\|_{K}^{2}\right) \leq\left(\frac{2 \mathcal{D}(\lambda)}{\lambda}+\frac{9 \lambda^{\max \{p-2,-p\}} T^{1-\theta}}{(1-\theta) 2^{1-\theta}}\right) \\
\quad \exp \left\{-\frac{\left(1-2^{\theta-1}\right) \lambda^{\max \{p, 1\}}}{(1-\theta) C_{\phi, \kappa}} T^{1-\theta}\right\}+\frac{19 C_{\phi, \kappa}}{\lambda^{\min \{p+1,2\}} T^{\theta}} .
\end{gathered}
$$

Proof: The increment condition (63) for $\phi_{-}^{\prime}$ tells us that

$$
\widetilde{C}_{\lambda} \leq 4 \kappa^{2} \max \left\{\left\|\phi_{-}^{\prime}\right\|_{L^{\infty}[-1,1]}, c_{p}\left(\kappa^{2}\left|\phi^{\prime}(0)\right| / \lambda\right)^{p}\right\}
$$

which implies

$$
\widetilde{C}_{\lambda} \leq C_{\phi, \kappa}^{2} \lambda^{-p}, \quad \forall \lambda \leq 1
$$

Also, the local Lipschitz constant can be bounded as

$$
\begin{gathered}
M(\lambda) \leq \max \left\{\left\|\frac{\phi_{-}^{\prime}(x)-\phi^{\prime}(0)}{x}\right\|_{L^{\infty}[-1,1]},\right. \\
\left.c_{p}\left(\kappa^{2}\left|\phi^{\prime}(0)\right| / \lambda\right)^{p-1}+\left|\phi^{\prime}(0)\right|\right\} .
\end{gathered}
$$

Choose $\mu(\lambda)=C_{\phi, \kappa} \lambda^{-\max \{p-1,0\}}$. Then

$$
M(\lambda) \kappa^{2}+\lambda \leq \mu(\lambda), \quad \forall \lambda \leq 1
$$

and our conclusion follows from Theorem 5 .

\section{TOTAL ERRor Bounds AND LEARNING RATES}

Applying the above mentioned techniques, we can derive the learning rates of the excess misclassification error for the online algorithm (4) from our analysis on $\left\|f_{T+1}-f_{\lambda}\right\|_{K}$ together with the regularization error $\mathcal{D}(\lambda)$.

\section{A. Rates for the online algorithm with the hinge loss}

First we prove the learning rates for the online algorithm (13) with the hinge loss.

Proof of Corollary 1: Consider the hinge loss $\phi(x)=$ $(1-x)_{+}$. Recall the relation (18) between the excess misclassification error and the excess generalization error. Then

$\mathcal{R}(\operatorname{sgn}(f))-\mathcal{R}\left(f_{c}\right) \leq \mathcal{E}(f)-\mathcal{E}\left(f_{c}\right) \leq \mathcal{E}(f)-\mathcal{E}\left(f_{\lambda}\right)+\mathcal{D}(\lambda)$.

Using the uniform Lipschitz continuity of the hinge loss, we know that $\mathcal{E}\left(f_{T+1}\right)-\mathcal{E}\left(f_{\lambda}\right) \leq\left\|f_{T+1}-f_{\lambda}\right\|_{L_{\rho_{X}}^{1}}$. Combined with the assumption on the regularization error $\mathcal{D}(\lambda) \leq c_{\beta} \lambda^{\beta}$ for some $c_{\beta}>0$, it follows from (66) that

$$
\begin{aligned}
& \mathcal{R}\left(\operatorname{sgn}\left(f_{T+1}\right)\right)-\mathcal{R}\left(f_{c}\right) \leq\left\|f_{T+1}-f_{\lambda}\right\|_{L_{\rho_{X}}^{1}}+c_{\beta} \lambda^{\beta} \\
& \leq \kappa\left\|f_{T+1}-f_{\lambda}\right\|_{K}+c_{\beta} \lambda^{\beta} .
\end{aligned}
$$

Now we apply Corollary 7. It is easy to see that $\phi$ satisfies (63) with $p=0, c_{p}=1$ and $C_{\phi, \kappa}=2+\kappa^{2}$. For any $0<$ $\varepsilon<\frac{\beta}{2(\beta+1)}$, choose $\theta=\frac{2 \beta+1}{2(\beta+1)}-\frac{(2 \beta+1) \varepsilon}{\beta}$ in Corollary 7. We know that $\mathbb{E}\left(\left\|f_{T+1}-f_{\lambda}\right\|_{K}^{2}\right)$ is bounded by

$$
\begin{aligned}
& {\left[\frac{2 \mathcal{D}(\lambda)}{\lambda}+\frac{9 T^{1-\theta}}{(1-\theta) 2^{1-\theta}}\right] \exp \left\{-\frac{\left(1-2^{\theta-1}\right) \lambda}{(1-\theta)\left(2+\kappa^{2}\right)} T^{1-\theta}\right\}} \\
& +\frac{19\left(2+\kappa^{2}\right)}{\lambda T^{\theta}} .
\end{aligned}
$$

Select $\lambda=T^{-\gamma}$ with $0<\gamma<\min (1-\theta, \theta)$. Since the asymptotic behavior $\exp \left\{-c T^{\epsilon}\right\}=O\left(T^{-s}\right)$ holds for any $\epsilon>0, s>0$ and $c>0$. We know that there exists a constant $C_{\theta, \gamma, \kappa}$ depending only on $\theta, \kappa$ and $\gamma$ such that

$$
\mathbb{E}\left(\left\|f_{T+1}-f_{\lambda}\right\|_{K}^{2}\right) \leq C_{\theta, \gamma, \kappa} T^{-\theta+\gamma} .
$$

Putting this back into (67), we have

$\mathbb{E}\left(\mathcal{R}\left(\operatorname{sgn}\left(f_{T+1}\right)\right)-\mathcal{R}\left(f_{c}\right)\right) \leq \kappa \sqrt{C_{\theta, \gamma, \kappa}} T^{-\frac{(\theta-\gamma)}{2}}+c_{\beta} T^{-\beta \gamma}$.

Now take $\gamma=\frac{1}{2(\beta+1)}-\frac{\varepsilon}{\beta}$. We know that the following holds

$$
\mathbb{E}\left(\mathcal{R}\left(\operatorname{sgn}\left(f_{T+1}\right)\right)-\mathcal{R}\left(f_{c}\right)\right)=O\left(T^{\varepsilon-\frac{\beta}{2(\beta+1)}}\right) .
$$

This proves our conclusion. 
B. Rates for the online algorithm with the least square loss

Turn to the least square loss. It has a special feature that $\left(\phi^{\prime}(x)\right)^{2}=4 \phi(x)$. This enables us to improve the learning rates.

Proof of Corollary 2: For the least square loss, we use the relation (see [13])

$$
\begin{aligned}
& \mathcal{R}(\operatorname{sgn}(f))-\mathcal{R}\left(f_{c}\right)=\int_{\operatorname{Sgn}(f) \neq \operatorname{sgn}\left(f_{\rho}\right)}\left|f_{\rho}(x)\right| d \rho_{X} \\
& \leq \int_{X}\left|f(x)-f_{\rho}(x)\right| d \rho_{X} \leq\left\|f-f_{\rho}\right\|_{L_{\rho_{X}}^{2}} .
\end{aligned}
$$

Then we can estimate the excess misclassification error $\mathcal{R}\left(\operatorname{sgn}\left(f_{T+1}\right)\right)-\mathcal{R}\left(f_{c}\right)$ can be bounded by

$$
\left\|f_{T+1}-f_{\lambda}\right\|_{L_{\rho_{X}}^{2}}+\left\|f_{\lambda}-f_{\rho}\right\|_{L_{\rho_{X}}^{2}} .
$$

We know from Lemma 3 in [26] that if $f_{\rho}$ is in the range of $L_{K}^{\beta}$ for some $0<\beta \leq 1$ then the second term of (68) can be bounded as

$$
\left\|f_{\lambda}-f_{\rho}\right\|_{L_{\rho_{X}}^{2}} \leq \lambda^{\beta}\left\|L_{K}^{-\beta} f_{\rho}\right\|_{L_{\rho_{X}}^{2}} .
$$

The first term $\left\|f_{T+1}-f_{\lambda}\right\|_{L_{\rho_{X}}^{2}} \leq \kappa\left\|f_{T+1}-f_{\lambda}\right\|_{K}$ can be estimated by a refined bound for $\mathbb{E}\left(\left\|f_{T+1}-f_{\lambda}\right\|_{K}^{2}\right)$. Namely, if $\mu(\lambda)=8 \kappa^{2}+\lambda$ and $\eta_{t} \mu(\lambda) \leq 1$ for each $t \in \mathbb{N}$, there holds

$$
\begin{array}{r}
\mathbb{E}\left(\left\|f_{T+1}-f_{\lambda}\right\|_{K}^{2}\right) \leq\left[\frac{2 \mathcal{D}(\lambda)}{\lambda}+\frac{144 \kappa^{2} T^{1-\theta}}{(1-\theta) 2^{1-\theta}(\mu(\lambda))^{2}}\right] \\
\exp \left\{-\frac{\left(1-2^{\theta-1}\right) \lambda}{2(1-\theta) \mu(\lambda)} T^{1-\theta}\right\}+\frac{608 \kappa^{2}}{\mu(\lambda) \lambda T^{\theta}} .
\end{array}
$$

Let us first prove (70). The choice of $\eta_{t}$ and Theorem 4 tell us that the learning sequence is uniformly bounded as

$$
\left\|f_{t}\right\|_{K} \leq 2 \kappa / \lambda \text {. }
$$

The special feature $\left(\phi^{\prime}(x)\right)^{2}=4 \phi(x)$ of the least square loss yields

$$
\begin{aligned}
& \mathbb{E}_{z_{1}, \ldots, z_{t}}\left(\left\|\phi_{-}^{\prime}\left(y_{t} f_{t}\left(x_{t}\right)\right) y_{t} K_{x_{t}}+\lambda f_{t}\right\|_{K}^{2}\right) \\
\leq & 2\left(\kappa^{2} \mathbb{E}_{z_{1}, \ldots, z_{t}}\left[\phi_{-}^{\prime}\left(y_{t} f_{t}\left(x_{t}\right)\right)\right]^{2}+4 \kappa^{2}\right) \\
\leq & 8 \kappa^{2}\left(\mathbb{E}_{z_{1}, \ldots, z_{t-1}}\left(\mathbb{E}_{z_{t}}\left[\phi\left(y_{t} f_{t}\left(x_{t}\right)\right)\right]\right)+1\right) \\
= & 8 \kappa^{2}\left(\mathbb{E}_{z_{1}, \ldots, z_{t-1}}\left(\mathcal{E}\left(f_{t}\right)\right)+1\right) .
\end{aligned}
$$

Combining with the estimate $\left.\mathcal{E}\left(f_{\lambda}\right)\right)+\frac{\lambda}{2}\left\|f_{\lambda}\right\|_{K}^{2} \leq \mathcal{E}(0)=1$, we know (71) is bounded by

$$
\begin{aligned}
8 \kappa^{2} & \left(\mathbb { E } _ { z _ { 1 } , \ldots , z _ { t - 1 } } \left[\left\{\mathcal{E}\left(f_{t}\right)+\frac{\lambda}{2}\left\|f_{t}\right\|_{K}^{2}\right\}\right.\right. \\
& \left.\left.-\left\{\mathcal{E}\left(f_{\lambda}\right)+\frac{\lambda}{2}\left\|f_{\lambda}\right\|_{K}^{2}\right\}\right]\right)+16 \kappa^{2} .
\end{aligned}
$$

The same procedure as (53) in the proof of Lemma 3 gives

$$
\begin{aligned}
& \mathbb{E}_{z_{1}, \ldots, z_{t}}\left(\left\|f_{t+1}-f_{\lambda}\right\|_{K}^{2}\right) \leq \mathbb{E}_{z_{1}, \ldots, z_{t-1}}\left(\left\|f_{t}-f_{\lambda}\right\|_{K}^{2}\right) \\
& +\eta_{t}^{2} \mathbb{E}_{z_{1}, \ldots, z_{t}}\left(\left\|\phi_{-}^{\prime}\left(y_{t} f_{t}\left(x_{t}\right)\right) y_{t} K_{x_{t}}+\lambda f_{t}\right\|_{K}^{2}\right) \\
& -2 \eta_{t} \mathbb{E}_{z_{1}, \ldots, z_{t-1}}\left[\left\{\mathcal{E}\left(f_{t}\right)+\frac{\lambda}{2}\left\|f_{t}\right\|_{K}^{2}\right\}\right. \\
& \left.\quad-\left\{\mathcal{E}\left(f_{\lambda}\right)+\frac{\lambda}{2}\left\|f_{\lambda}\right\|_{K}^{2}\right\}\right] .
\end{aligned}
$$

This combining with (71) and (72) implies

$$
\begin{gathered}
\mathbb{E}_{z_{1}, \ldots, z_{t}}\left(\left\|f_{t+1}-f_{\lambda}\right\|_{K}^{2}\right) \leq \mathbb{E}_{z_{1}, \ldots, z_{t-1}}\left(\left\|f_{t}-f_{\lambda}\right\|_{K}^{2}\right) \\
+16 \kappa^{2} \eta_{t}^{2}-2 \eta_{t}\left(1-4 \kappa^{2} \eta_{t}\right) \mathbb{E}_{z_{1}, \ldots, z_{t-1}}\left[\left\{\mathcal{E}\left(f_{t}\right)\right.\right. \\
\left.\left.+\frac{\lambda}{2}\left\|f_{t}\right\|_{K}^{2}\right\}-\left\{\mathcal{E}\left(f_{\lambda}\right)+\frac{\lambda}{2}\left\|f_{\lambda}\right\|_{K}^{2}\right\}\right] .
\end{gathered}
$$

From this bound, Theorem 2 and the restriction $4 \kappa^{2} \eta_{t} \leq 1 / 2$, we know that $\mathbb{E}_{z_{1}, \ldots, z_{t}}\left(\left\|f_{t+1}-f_{\lambda}\right\|_{K}^{2}\right)$ can be bounded by

$$
\left(1-\frac{\eta_{t} \lambda}{2}\right) \mathbb{E}_{z_{1}, \ldots, z_{t-1}}\left(\left\|f_{t}-f_{\lambda}\right\|_{K}^{2}\right)+64 \kappa^{2}\left(\eta_{t} / 2\right)^{2} .
$$

Applying this relation iteratively for $t=T, \ldots, 1$, we see that

$$
\begin{aligned}
& \mathbb{E}\left(\left\|f_{T+1}-f_{\lambda}\right\|_{K}^{2}\right) \leq \exp \left\{-\frac{\lambda}{2 \mu(\lambda)} \sum_{t=1}^{T} t^{-\theta}\right\}\left\|f_{\lambda}\right\|_{K}^{2} \\
& +\frac{64 \kappa^{2}}{(2 \mu(\lambda))^{2}} \sum_{t=1}^{T} t^{-2 \theta} \exp \left\{-\frac{\lambda}{(2 \mu(\lambda))} \sum_{j=t+1}^{T} j^{-\theta}\right\} .
\end{aligned}
$$

By Lemma 5 with $0<\theta<1$, we know that

$$
\begin{aligned}
& \mathbb{E}\left(\left\|f_{T+1}-f_{\lambda}\right\|_{K}^{2}\right) \leq\left[\frac{2 \mathcal{D}(\lambda)}{\lambda}+\frac{144 \kappa^{2} T^{1-\theta}}{(1-\theta) 2^{1-\theta}(\mu(\lambda))^{2}}\right] \\
& \exp \left\{-\frac{\left(1-2^{\theta-1}\right) \lambda}{2(1-\theta) \mu(\lambda)} T^{1-\theta}\right\}+\frac{608 \kappa^{2}}{\mu(\lambda) \lambda T^{\theta}}
\end{aligned}
$$

which verifies (70).

Choose $\lambda=T^{-\gamma}$ with $0<\gamma<1-\theta$. For some constant $C_{\theta, \gamma, \kappa}$, we have

$$
\mathbb{E}\left(\left\|f_{T+1}-f_{\lambda}\right\|_{K}^{2}\right) \leq C_{\theta, \gamma, \kappa} T^{-(\theta-\gamma)} .
$$

Thus, $\mathbb{E}\left(\left\|f_{T+1}-f_{\lambda}\right\|_{L_{\rho_{X}}^{2}}\right)$ is bounded by

$$
\kappa \mathbb{E}\left(\left\|f_{T+1}-f_{\lambda}\right\|_{K}\right) \leq \kappa \sqrt{C_{\theta, \gamma, \kappa}} T^{-(\theta-\gamma) / 2} .
$$
have

Combining with (68) and (69), for a constant $C_{\gamma, \beta, \kappa, \theta}^{\prime}$ we

$$
\mathbb{E}\left(\left\|f_{T+1}-f_{\rho}\right\|_{L_{\rho_{X}}^{2}}\right) \leq C_{\gamma, \beta, \kappa, \theta}^{\prime}\left(T^{-(\theta-\gamma) / 2}+T^{-\beta \gamma}\right) .
$$

Choose $\theta=\frac{2 \beta+1}{2(\beta+1)}-\frac{(2 \beta+1) \varepsilon}{\beta}$ and $\gamma=\theta /(2 \beta+1)$. We obtain the desired bound

$$
\mathbb{E}\left(\left\|f_{T+1}-f_{\rho}\right\|_{L_{\rho_{X}}^{2}}\right) \leq C_{\varepsilon, \kappa, \beta} T^{\varepsilon-\frac{\beta}{2(\beta+1)}} .
$$

The proof of the corollary is completed.

Proof of Theorem 3: If the regression function $f_{\rho}$ is in the range of $L_{K}^{\beta}$ for $1 / 2<\beta \leq 1$, then by Lemma 3 in [26] we know that

$$
\left\|f_{\lambda}-f_{\rho}\right\|_{K} \leq \lambda^{\beta-1 / 2}\left\|L_{K}^{-\beta} f_{\rho}\right\|_{L_{\rho_{X}}^{2}}
$$

Replacing $\beta$ with $\beta-1 / 2$ and arguing as in the proof of corollary 2 , we obtain the desired result. 


\section{Rates with the q-norm SVM loss}

We shall use the relation (19) to derive the learning rate. To estimate $\mathcal{E}\left(f_{T+1}\right)-\mathcal{E}\left(f_{\lambda}\right)$ using the error in the $\mathcal{H}_{K}$ metric, we need the following lemma.

Lemma 6: For the $q$-norm SVM loss $\phi(x)=(1-x)_{+}^{q}$ with $q>1$, and $f \in \mathcal{H}_{K}$, there holds

$$
\begin{gathered}
\mathcal{E}(f)-\mathcal{E}\left(f_{\lambda}\right) \leq q \kappa[2(1+\kappa)]^{q-1} \\
{\left[(1+\sqrt{2 \mathcal{D}(\lambda) / \lambda})^{q-1}\left\|f-f_{\lambda}\right\|_{K}+\left\|f-f_{\lambda}\right\|_{K}^{q}\right] .}
\end{gathered}
$$

Proof: Note the inequality [7]

$$
\begin{aligned}
\left|(1-u)_{+}^{q}-(1-v)_{+}^{q}\right| & \leq q(1+\max (|u|,|v|))^{q-1}|u-v| \\
& \leq q(1+|u-v|+|v|)^{q-1}|u-v| .
\end{aligned}
$$

It follows by taking $u=y f(x), v=y f_{\lambda}(x)$ that $\mathcal{E}(f)-\mathcal{E}\left(f_{\lambda}\right)$ can be bounded by

$$
\begin{array}{ll} 
& q \kappa\left(\left[1+\kappa\left\|f-f_{\lambda}\right\|_{K}+\kappa\left\|f_{\lambda}\right\|_{K}\right]^{q-1}\left\|f-f_{\lambda}\right\|_{K}\right) \\
\leq \quad & q \kappa\left(\left[1+\kappa\left\|f-f_{\lambda}\right\|_{K}+\kappa \sqrt{2 \mathcal{D}(\lambda) / \lambda}\right]^{q-1}\left\|f-f_{\lambda}\right\|_{K}\right) \\
\leq \quad & q \kappa[2(1+\kappa)]^{q-1}\left[(1+\sqrt{2 \mathcal{D}(\lambda) / \lambda})^{q-1}\left\|f-f_{\lambda}\right\|_{K}\right. \\
& \left.+\left\|f-f_{\lambda}\right\|_{K}^{q}\right]
\end{array}
$$

where the bound (24) for $f_{\lambda}$ is used. This completes the proof of the lemma.

Proof of Corollary 4: We apply Corollary 7. Observe that $\phi(x)=(1-x)_{+}^{q}$ satisfies (63) with $c_{p}=q 2^{q-1}, p=$ $q-1$ and $C_{\phi, \kappa}=\left(1+2 \kappa^{2 q}\right)(2 q)^{q+1}$. Choose $0<\gamma<$ $\min \left\{\frac{1-\theta}{\max \{q-1,1\}}, \frac{\theta}{\min \{q, 2\}}\right\}$ and $\lambda=T^{-\gamma}$. It follows from (65) that for a constant $C_{q, \kappa, \theta, \gamma}$ depending only on $q, \kappa, \theta$, and $\gamma$ such that

$$
\mathbb{E}\left(\left\|f_{T+1}-f_{\lambda}\right\|_{K}^{2}\right) \leq C_{q, \kappa, \theta, \gamma} T^{-(\theta-\min \{q, 2\} \gamma)} .
$$

This in connection with (73) and the assumption on the regularization error $\mathcal{D}(\lambda) \leq c_{\beta} \lambda^{\beta}$ that $\mathcal{E}\left(f_{T+1}\right)-\mathcal{E}\left(f_{\lambda}\right)$ can be bounded by

$$
C_{q, \kappa, \beta}\left[\lambda^{(\beta-1)(q-1) / 2}\left\|f_{T+1}-f_{\lambda}\right\|_{K}+\left\|f_{T+1}-f_{\lambda}\right\|_{K}^{q}\right]
$$

where $C_{q, \kappa, \beta}$ is a constant depending on $q, \kappa$, and $\beta$. Thus, with another constant $\widetilde{C}_{q, \kappa, \gamma, \beta}, \mathbb{E}\left(\mathcal{E}\left(f_{T+1}\right)-\mathcal{E}\left(f_{\lambda}\right)\right)$ can be bounded by

$$
\widetilde{C}_{q, \kappa, \gamma, \beta} T^{(1-\beta)(q-1) \gamma / 2} T^{-(\theta-\min (q, 2) \gamma) / 2} .
$$

Combining with the error decomposition (22) and the regularization error decay, this implies that $\mathbb{E}\left(\mathcal{E}\left(f_{T+1}\right)-\mathcal{E}\left(f_{\rho}^{\phi}\right)\right)$ is bounded by

$$
\widetilde{C}_{q, \gamma, \kappa, \beta}\left(T^{-(\theta-[\min \{q-1,1\}+(1-\beta) q+\beta] \gamma) / 2}+T^{-\beta \gamma}\right) .
$$

Choose

$$
\begin{aligned}
\theta= & \frac{\min \{q-1,1\}+(1-\beta) q+3 \beta}{(2-\beta) q+3 \beta} \\
& -\frac{2[\min \{q-1,1\}+(1-\beta) q+3 \beta] \varepsilon}{\beta} \\
= & {[\min \{q-1,1\}+(1-\beta) q+3 \beta] \gamma, }
\end{aligned}
$$

then the desired result follows by the comparison relations (19) and (21).

\section{CONCLUSION AND QUESTIONS}

In this paper we have investigated the strong convergence of the online regularized classification algorithm involving general loss functions and reproducing kernel Hilbert spaces. We verified the convergence under rather weak assumptions on the step sizes and loss functions. A novel relation between the error in the RKHS norm and the excess regularized generalization error played an important role. As done for the off-line setting in the literature, by suitable choices of the regularization parameter $\lambda=\lambda(T)$ according to a priori conditions on the approximation error, we presented explicit capacity independent learning rates of the excess misclassification error for commonly used loss functions such as the hinge loss, the least square loss, and the SVM $q$-norm loss. In particular, we have shown (remarks following Theorem 3) that our learning rates in $\mathcal{H}_{K}$ with the least square loss are comparable to the ones in the off-line setting.

Let us mention some questions for further study.

1. In this paper the regularization parameter $\lambda=\lambda(T)$ depends only on the sample size $T$ which means that (4) studied here is not a fully online algorithm. How to analyze the algorithm (4) with $\lambda$ changing with the steps, $\lambda=\lambda(t)$, remains open.

2. It would be interesting to analyze the online algorithm in the case $\lambda=0$. This is closely related to the perceptron algorithms (see [29], [10]).

3. Here we assume the data $\mathbf{z}=\left\{z_{t}\right\}_{j=1}^{T}$ is i.i.d. according to an unknown distribution $\rho$. In many applications the data is not independent or identical. It's unknown whether Markov chains and the theory of martingales can be used to deal with this case.

\section{ACKNOWLEDGMENT}

The authors would like to thank the referees for valuable comments and suggestions.

\section{APPENDIX}

\section{Online Algorithm as Descent Method}

The classical descent method [6] is an efficient method to solve unconstrained minimization problems of the form

$$
x^{*}=\arg \min _{x \in \mathbb{R}^{n}} F(x)
$$

where $F: \mathbb{R}^{n} \rightarrow \mathbb{R}$ is convex and continuously differentiable. The descent method is to find a suitable sequence $x^{k}, k=$ $1,2, \ldots$ to approximate the minimum point $x^{*}$ :

$$
x^{k+1}=x^{k}+\eta_{k} \Delta x^{k}
$$

where $1>\eta_{k}>0$ is the step size and $\Delta x^{k}$ denotes the descent direction.

The descent method requires that

$$
F\left(x^{k+1}\right)<F\left(x^{k}\right)
$$

except when $x^{k}$ is optimal. The Taylor expansion of $F$ tells us

$$
F\left(x^{k+1}\right)=F\left(x^{k}\right)+\eta_{k} \nabla F\left(x^{k}\right) \cdot \triangle x^{k}+o\left(\eta_{k}\left|\triangle x^{k}\right|\right)
$$


which requires

$$
\nabla F\left(x^{k}\right) \cdot \triangle x^{k}<0
$$

That is, the direction $\triangle x^{k}$ must make an obtuse angle with the gradient $\nabla F\left(x^{k}\right)$. If we select the descent direction $\triangle x_{k}$ as the negative gradient direction, this gives the well known descent algorithm

$$
x^{k+1}=x^{k}-\eta_{k} \nabla F\left(x^{k}\right) .
$$

Under some assumptions on the step sizes $\eta_{k}$, one can get the convergence [6]

$$
F\left(x^{k}\right) \rightarrow F\left(x^{*}\right)
$$

The above descent method motivates the classification algorithm (4). To see this, let us assume that the classifying loss $\phi$ is differentiable. Since the regularizing function $f_{\lambda}$ is an unconstrained minimizer in $\mathcal{H}_{K}$ of the functional (the regularized generalization error)

$$
F(f)=\mathcal{E}(f)+\frac{\lambda}{2}\|f\|_{K}^{2},
$$

as the descent algorithm, we use the following sequence $\left\{g_{t}\right\}_{t=1}^{T} \subset \mathcal{H}_{K}$ to approximate $f_{\lambda}$

$$
g_{t+1}=g_{t}-\left.\eta_{t} \partial\left(\mathcal{E}(f)+\frac{\lambda}{2}\|f\|_{K}^{2}\right)\right|_{f=g_{t}} \quad \text { with } g_{1}=0 .
$$

Note that the functional derivative (see [33])

$$
\left.\partial\left(\mathcal{E}(f)+\frac{\lambda}{2}\|f\|_{K}^{2}\right)\right|_{f=g_{t}}=\int_{Z} \phi^{\prime}\left(y g_{t}(x)\right) y K_{x} d \rho+\lambda g_{t} .
$$

However, the distribution $\rho$ is unknown in practical classification problems. What we have is a random sample $\mathbf{z}=\left\{z_{t}=\left(x_{t}, y_{t}\right)\right\}_{t=1}^{T}$. So we replace the integral part $\int_{Z} \phi^{\prime}(y f(x)) y K_{x} d \rho$ by the random value $\phi^{\prime}\left(y_{t} f\left(x_{t}\right)\right) y_{t} K_{x_{t}}$, and the above descent scheme then becomes the stochastic gradient descent online algorithm (4).

\section{REFERENCES}

[1] M. Anthony and P. L. Bartlett, Neural Network Learning: Theoretical Foundations, Cambridge University Press, 1999.

[2] N. Aronszajn, Theory of reproducing kernels, Trans. Amer. Math. Soc. 68 (1950), 337-404.

[3] K. S. Azoury and M. K. Warmuth, Relative loss bounds for on-line density estimation with the exponential family of distributions, Machine Learning 43 (2001), 211-246.

[4] P. L. Bartlett, M. I. Jordan and J. D. McAuliffe, Convexity, classification, and risk bounds, J. Amer. Statist. Assoc. 101 (2006), 138-156.

[5] O. Bousquet and A. Elisseeff, Stability and generalization, J. Machine Learning Res. 2 (2002), 499-526.

[6] S. Boyd and L. Vandenberghe, Convex optimization, Cambridge, 2004.

[7] D. R. Chen, Q. Wu, Y. Ying and D. X. Zhou, Support vector machine soft margin classifiers: error analysis, J. Machine Learning Res. 5 (2004), $1143-1175$.

[8] N. Cesa-Bianchi, A. Conconi and C. Gentile, A second-order perceptron algorithm, SIAM J. Comput. 34 (2005), 640-688.

[9] N. Cesa-Bianchi, P. Long and M. K. Warmuth, Worst-case quadratic loss bounds for prediction using linear functions and gradient descent, IEEE Trans. Neural Networks 7 (1996), 604-619.

[10] N. Cesa-Bianchi, A. Conconi and C. Gentile, On the generalization ability of on-line learning algorithms, IEEE Trans. Inform. Theory $\mathbf{5 0}$ (2004), 2050-2057.

[11] F. Cucker and S. Smale, On the mathematical foundations of learning, Bull. Amer. Math. Soc. 39 (2001), 1-49.

[12] E. De Vito, A. Caponnetto, and L. Rosasco, Model selection for regularized least-squares algorithm in learning theory, Found. Comput. Math. 5 (2005), 59-85.
[13] L. Devroye, L. Györfi and G. Lugosi, A Probabilistic Theory of Pattern Recognition, Springer-Verlag, New York, 1997.

[14] T. Evgeniou, M. Pontil and T. Poggio, Regularization networks and support vector machines, Adv. Comput. Math. 13 (2000), 1-50.

[15] J. Forster and M. K. Warmuth, Relative expected instantaneous loss bounds, J. Computer and System Sciences, 64 (2002), 76-102.

[16] M. Herbster and M. K. Warmuth, Tracking the best expert, Machine Learning 32 (1998), 151-178.

[17] J. Kivinen, A. J. Smola and R. C. Williamson, Online learning with kernels, IEEE Trans. Signal Processing 52 (2004), 2165-2176.

[18] Y. Lin, Support vector machines and the Bayes rule in classification, Data Mining and Knowledge Discovery 6 (2002), 259-275.

[19] G. Lugosi and N. Vayatis, On the Bayes-risk consistency of regularized boosting methods, Ann. Stat. 32 (2004), 30-55.

[20] P. Niyogi and F. Girosi, On the relationships between generalization error, hypothesis complexity and sample complexity for radial basis functions, Neural Comp. 8 (1996), 819-842.

[21] C. Scovel and I. Steinwart, Fast rates for support vector machines, in Proceedings of the Conference on Learning Theory (COLT-2005), pp. 279-294.

[22] S. Smale and Y. Yao, Online learning algorithms, Found. Comp. Math. 6 (2006), 145-170.

[23] S. Smale and D.X. Zhou, Estimating the approximation error in learning theory, Anal. Appl. 1 (2003), 17-41.

[24] S. Smale and D.X. Zhou, Shannon sampling and function reconstruction from point values, Bull. Amer. Math. Soc. 41 (2004), 279-305.

[25] S. Smale and D.X. Zhou, Shannon sampling II: Connection to learning theory, Appl. Comput. Harmonic Anal. 19 (2005), 285-302.

[26] S. Smale and D.X. Zhou, Learning theory estimates via integral operators and their applications, Constr. Approx., to appear.

[27] I. Steinwart, Support vector machines are universally consistent, $J$. Complexity 18 (2002), 768-791.

[28] A. B. Tsybakov, Optimal aggregation of classifiers in statistical learning, Ann. Stat. 32 (2004), 135-166.

[29] V. Vapnik, Statistical Learning Theory, John Wiley \& Sons, 1998.

[30] G. Wahba, Spline models for observational data, SIAM, 1990.

[31] Q. Wu, Y. Ying and D. X. Zhou, Multi-kernel Regularized Classifiers, J. Complexity, in press.

[32] Q. Wu and D. X. Zhou, SVM soft margin classifiers: linear programming versus quadratic programming, Neural Comp. 17 (2005), 1160-1187.

[33] K. Yosida, Functional analysis, 6th edition, Springer-Verlag, 1980.

[34] Y. Ying and D.X. Zhou, Learnability of Gaussians with flexible variances, preprint, 2004.

[35] T. Zhang, Statistical behavior and consistency of classification methods based on convex risk minimization, Ann. Stat. 32 (2004), 56-85.

[36] T. Zhang, Leave-one-out bounds for kernel methods, Neural Comp. 15 (2003), 1397-1437.

[37] D. X. Zhou, The covering number in learning theory, J. Complexity 18 (2002), 739-767.

[38] D. X. Zhou, Capacity of reproducing kernel spaces in learning theory, IEEE Trans. Inform. Theory 49 (2003), 1743-1752.

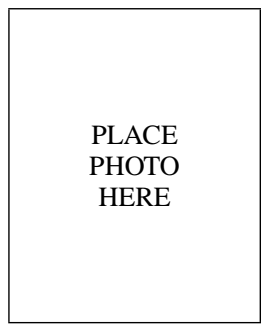

Yiming Ying was born in Zhejiang, China in 1976. $\mathrm{He}$ received the B.S. degree in mathematics from Hangzhou University in 1997, Hangzhou, China and the Ph.D. degree in mathematics from Zhejiang University in 2002, Hangzhou, China.

In 2002 he was a postdoctor at the Institute of Mathematics, Academia Sinica, Beijing, China. From 2003 to July 2005 he was a Research Fellow at the City University of Hong Kong, Hong Kong, China. He is currently a postdoctoral Research Fellow at the Department of Computer Science, University College London, London, UK. His research interests include harmonic analysis, learning theory, and machine learning.

Ding-Xuan Zhou was born in Zhejiang, China in 1967. He received the B.S. and Ph.D. degrees in mathematics in 1988 and 1991, respectively, from Zhejiang University, Hangzhou, China 
In 1992 he was a postdoctor at the Institute of Mathematics, Academia Sinica, Beijing, China. From 1993 to July 1995 he was a Humboldt Research Fellow at the Universität Duisburg, Germany. He was a Postdoctoral Fellow and Instructor at the University of Alberta, Edmonton, Canada, from August 1995 to November 1996. In the winter of 1996, he joined the faculty of City University of Hong Kong, where he is currently Professor and Head of the Department of Mathematics. His research interests include learning theory, approximation theory, and wavelet analysis. 